\title{
Spin Foam Models
}

\author{
John C. Baez \\ Department of Mathematics, University of California \\ Riverside, California 92521 \\ USA
}

email: baez@math.ucr.edu

September 19, 1997

\begin{abstract}
While the use of spin networks has greatly improved our understanding of the kinematical aspects of quantum gravity, the dynamical aspects remain obscure. To address this problem, we define the concept of a 'spin foam' going from one spin network to another. Just as a spin network is a graph with edges labeled by representations and vertices labeled by intertwining operators, a spin foam is a 2-dimensional complex with faces labeled by representations and edges labeled by intertwining operators. Spin foams arise naturally as higher-dimensional analogs of Feynman diagrams in quantum gravity and other gauge theories in the continuum, as well as in lattice gauge theory. When formulated as a 'spin foam model', such a theory consists of a rule for computing amplitudes from spin foam vertices, faces, and edges. The product of these amplitudes gives the amplitude for the spin foam, and the transition amplitude between spin networks is given as a sum over spin foams. After reviewing how spin networks describe 'quantum 3-geometries', we describe how spin foams describe 'quantum 4-geometries'. We conclude by presenting a spin foam model of 4-dimensional Euclidean quantum gravity, closely related to the state sum model of Barrett and Crane, but not assuming the presence of an underlying spacetime manifold.
\end{abstract}

\section{Introduction}

Thanks in part to the introduction of spin network techniques [55], we now have a mathematically rigorous and intuitively compelling picture of the kinematical aspects of what used to be called the 'loop representation' of quantum gravity [54]. Indeed, since spin networks form a very convenient basis of kinematical states [13, they have largely replaced collections of loops as our basic picture of 'quantum 3-geometries'. Unfortunately, the dynamical aspects of quantum gravity remain much less well understood in this approach. Over and above the many technical problems, the crucial fact is that we lack a simple picture of 'quantum 4-geometries'. However, recent work by various authors 32, 48, 51] suggests such a picture, which we formalize and explore here using the notion of a 'spin foam'.

The basic idea is simple. Just as a spin network is a graph with edges labeled by spins and vertices labeled by intertwining operators, a 'spin foam' is a 2-dimensional 
piecewise linear cell complex — roughly, a finite collection of polygons attached to each other along their edges — with faces labeled by spins and edges labeled by intertwining operators. As with spin networks, we may think of spin foams either abstractly or embedded in spacetime. Either way, a generic slice of a spin foam 'at fixed time' gives a spin network. Edges of this spin network come from faces of the spin foam, while vertices of the spin network come from edges of the spin foam. As we move the slice 'forwards in time', the spin network changes topology only when the slice passes a vertex of the spin foam.

In their joint work, Reisenberger and Rovelli [51] arrive at spin foams through the study of quantum gravity on a manifold of the form $\mathbb{R} \times S$ for some 3-manifold $S$ representing space. They begin with the Hamiltonian constraint $H$ with constant lapse function as an operator on the space of kinematical states. Of course, the actual form of this operator (if indeed it exists) is currently a matter of controversy: Thiemann has proposed a formula [59], but it is far from universally accepted. Luckily, Reisenberger and Rovelli's argument depends only on some general assumptions as to the nature of the operator $H$. In particular, they assume it generates a one-parameter group $\exp (-i t H)$. Since $H$ is a Hamiltonian constraint rather than a Hamiltonian, the physical interpretation of $\exp (-i t H)$ is somewhat problematic. Nonetheless, they argue that it is an object of substantial interest, encoding much of the dynamics of quantum gravity.

Under some assumptions on the form of the Hamiltonian constraint, Reisenberger and Rovelli are able to compute the transition amplitude $\langle\Psi, \exp (-i t H) \Phi\rangle$ as a formal power series in $t$ for any spin network states $\Psi$ and $\Phi$. The coefficient of $t^{n}$ in this power series is a sum over certain equivalence classes of spin foams embedded in spacetime with $\Psi$ as their initial slice, $\Phi$ as their final slice, and a total of $n$ foam vertices. Each spin foam contributes an amplitude given by a product over its vertices of certain 'spin foam vertex amplitudes'. In models with 'crossing symmetry', the spin foam vertex amplitude can be computed using only the spin network obtained by intersecting the spin foam with a small sphere about the vertex, and result depends only on the isotopy type of this spin network. This is to be expected in Euclidean quantum gravity, since it amounts to saying that applying a 4-dimensional rotation to a spin foam vertex does not affect its amplitude. In what follows we only consider models with crossing symmetry. (Unfortunately, Thiemann's formula for the Hamiltonian constraint lacks crossing symmetry.)

In this context, spin foams play a role much like that of Feynman diagrams (see Figure 1). In standard quantum field theory we compute transition amplitudes as sums or integrals over graphs with edges labeled by irreducible unitary representations of the relevant symmetry group. Typically this group is the product of the Poincaré group and some internal symmetry group, so the edges are labeled by momenta, spins, and certain internal quantum numbers. To compute the transition amplitude from one basis state to another, we sum over graphs going from one set of points labeled by representations (and vectors lying in these representations) to some other 
such set. The contribution of any graph to the amplitude is given by a product of amplitudes associated to its vertices and edges. Each vertex amplitude depends only on the representations labeling the incident edges, while each edge amplitude, or propagator, depends only on the label of the edge itself. The propagators are usually computed using a free theory about which we are doing a perturbative expansion, while the vertices represent interactions.
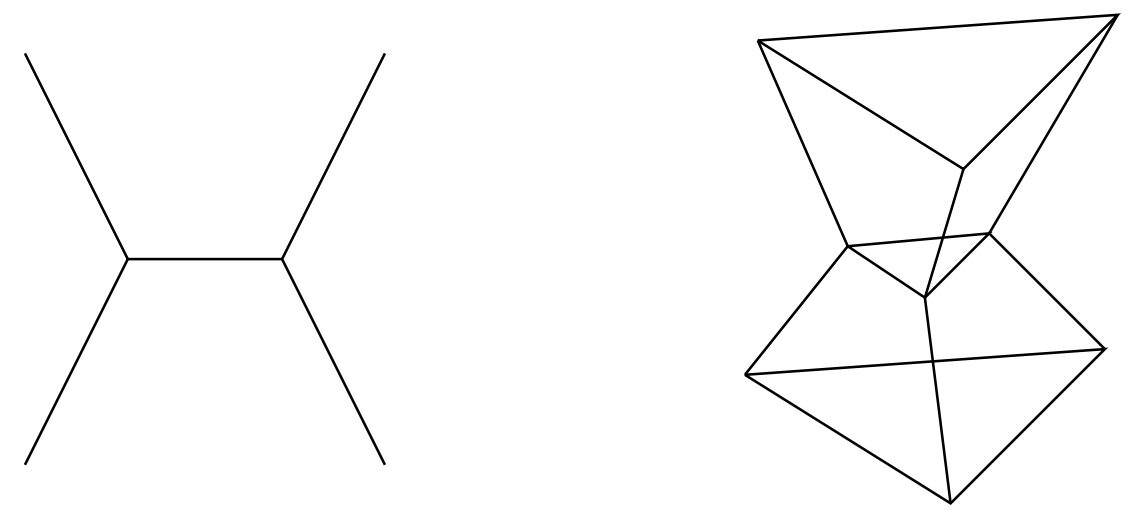

1. Feynman diagram versus spin foam

Similarly, we can consider 'spin foam models' for an arbitrary symmetry group $G$, generalizing the case considered by Reisenberger and Rovelli, in which $G=\mathrm{SU}(2)$. In this more general context a spin network is defined as a graph with oriented edges labeled by irreducible unitary representations of $G$ and vertices labeled by intertwining operators from the tensor product of the representations labeling 'incoming' incident edges to the tensor product of representations labeling 'outgoing' edges. By analogy, we define a spin foam to be a 2-dimensional piecewise linear cell complex with oriented faces labeled by irreducible unitary representations of $G$ and oriented edges labeled by intertwining operators.

In such a model we compute the transition amplitude between two spin networks as a sum over spin foams going from the first spin network to the second. Each spin foam contributes to the amplitude an amount given by a product of amplitudes associated to its vertices, edges, and faces. In a model with crossing symmetry, the amplitude of any vertex depends only on the isotopy class of the spin network obtained by intersecting the spin foam with a small sphere centered at the vertex. The amplitude of any edge depends only on the intertwining operator labeling that edge and the representations labeling the incident faces. The amplitude of any face depends only on the representation labeling that face. By analogy with Feynman diagrams, the edge and face amplitudes can be thought of as 'propagators'. Spin foam vertices can be thought of as 'interactions', and the vertex amplitudes characterize the nontrivial dynamics of the theory.

Reisenberger and Rovelli's approach to spin foams starting from canonical quantum gravity raises a number of problems: the well-definedness of the Hamiltonian 
constraint, the physical meaning of the one-parameter group $\exp (-i t H)$, the mathematical sense in which it can be approximated by a power series in $t$, and so on. It is reassuring therefore that spin foams also arise naturally in lattice gauge theory, where these problems are absent. The basic idea, due to Iwasaki [32] and Reisenberger [48], is that a path integral in lattice gauge theory can be represented as a sum over spin foams living in the lattice. The advantage of the lattice context is that it avoids some of the deep issues of analysis involved in the usual path-integral approach to quantum gravity. One can then address these issues by studying the limit in which the lattice is subdivided ever more finely.

Finally, in addition to canonical quantum gravity and lattice quantum gravity, it is worth comparing another approach to quantum gravity in which transition amplitudes can be computed using 2-dimensional analogs of Feynman diagrams: perturbative string theory. There are two major differences between spin foam models and perturbative string theory. First, perturbative string theory considers only 2manifolds with boundary mapped into spacetime, not more general piecewise linear cell complexes. Second, transition amplitudes in perturbative string theory are computed with the aid of a fixed metric on the ambient spacetime, while spin foam models make no reference to such a metric. These two facts are closely related. Since strings do not interact when they intersect in perturbative string theory, interactions arise primarily from the coupling of the string to the fixed metric on the ambient spacetime. In spin foam models, interactions occur where spin foam faces meet at vertices, so there is no need for a fixed background metric.

Interestingly, recent work on nonperturbative string theory suggests that strings and other higher-dimensional membranes interact where they intersect. Historically speaking, string theory and the loop representation of quantum gravity are twins separated at birth. Both arose from the failure of string theory as a viable fundamental theory of hadrons and the resulting attempts to reconcile its phenomenological successes with quantum chromodynamics. The two theories went very different ways, but they are still related. The author has already argued that the loop representation of quantum gravity can be viewed as a novel sort of background-free string theory [10], and shown that we may naturally associate a Wess-Zumino-Witten model to the surfaces appearing in the spacetime formulation of the loop representation of quantum gravity [16]. One reason for studying spin foams is to understand this better.

The plan of the paper is as follows. In Section 1 we give a precise definition of spin foams. It turns out that there is a category whose objects are spin networks and whose morphisms are spin foams. The basic idea is that a morphism $F: \Psi \rightarrow \Psi^{\prime}$ in this category is a spin foam $F$ going from the spin network $\Psi$ to the spin network $\Psi^{\prime}$. We can glue a spin foam $F: \Psi \rightarrow \Psi^{\prime}$ to a spin foam $F^{\prime}: \Psi^{\prime} \rightarrow \Psi^{\prime \prime}$ and obtain a spin foam $F F^{\prime}: \Psi \rightarrow \Psi^{\prime \prime}$. This sets up a useful analogy: 'spin foams are to operators as spin networks are to states'.

In addition to these 'abstract' spin foams one can consider spin foams embedded in a spacetime manifold. Various sorts of embeddings are interesting, with continuous, 
piecewise-linear, smooth and real-analytic ones being the most important. To keep this paper from becoming too long we discuss only one case here: piecewise-linearly embedded spin foams. The advantages of piecewise-linearly embedded spin networks have been pointed out by Zapata [64], the main one being the simplicity of a purely combinatorial approach. The same advantages apply when studying spin foams, but we emphasize that all options should be explored at this point. In Section 2 we sketch the role of piecewise-linearly embedded spin foams in lattice gauge theory.

In Section 3 we review the interpretation of spin networks as quantum 3-geometries. We work with $\mathrm{SU}(2)$ spin networks embedded in the dual 1-skeleton of a triangulated 3 -manifold $S$. In other words, we consider only spin networks having one 4 -valent vertex at the center of each tetrahedron in $S$ and one edge intersecting each triangle in $S$. For $\mathrm{SU}(2)$, irreducible representations are described by spins $j=0, \frac{1}{2}, 1, \ldots$, and an intertwining operator at a 4 -valent vertex can also be described by a spin. Thus we may work in the 'dual picture' and think of a spin network state as a labeling of all the triangles and tetrahedra in $S$ by spins [28, 31, 38, 53]. Thanks in part to Barbieri's work on the quantum tetrahedron [18], the Hilbert space spanned by these states can be interpreted as the space of 'quantum 3-geometries' of the triangulated 3-manifold $S$. In particular, one can define an 'area operator' for each triangle and a 'volume operator' for each tetrahedron. These operators are closely related to the area and volume operators first introduced by Rovelli and Smolin [56], as well as the slightly different ones studied by Ashtekar, Lewandowski and collaborators [6, 7, 8, 9, 33.

In Section 4 we turn to the interpretation of spin foams as quantum 4-geometries. Reisenberger and Rovelli [51] have already considered this issue for real-analytically embedded spin foams with gauge group SU(2). Here we use Spin(4) spin foams embedded in the dual 2-skeleton of a triangulated 4-manifold $M$. Such spin foams have one vertex centered at each 4 -simplex in $M$, one edge intersecting each tetrahedron, and one face intersecting each triangle. Since

$$
\operatorname{Spin}(4) \cong \mathrm{SU}(2) \times \mathrm{SU}(2),
$$

we can describe such a spin foam in the dual picture by labeling each triangle and each tetrahedron in $M$ by a pair of spins. Restricting attention to the 'left-handed' copy of $\mathrm{SU}(2)$, such a spin foam determines an $\mathrm{SU}(2)$ spin network in any 3-dimensional submanifold $S \subseteq M$ compatible with the triangulation. Thus, by the results described in the previous section, the spin foam endows each such submanifold with a quantum 3-geometry. However, for these quantum 3-geometries to fit together to form a sensible quantum 4-geometry, it appears that certain constraints must hold.

Following the ideas of Barrett and Crane [19], we arrive at these constraints through a study of the 'quantum 4-simplex'. Here we take $S$ to be the boundary of a single 4-simplex in $M$. A spin foam in $M$ gives each of the ten triangles in $S$ an area and each of the five tetrahedra in $S$ a volume. However, the geometry of a 4-simplex affinely embedded in Euclidean $\mathbb{R}^{4}$ is determined by only ten numbers, e.g., the lengths of its edges. This suggests that some constraints must hold for $S$ to be 
the boundary of a 'flat' 4 -simplex. We wish the 4 -simplices in $M$ to be flat because we want a picture similar to that of the Regge calculus, where spacetime is pieced together from flat 4-simplices, and curvature is concentrated along their boundaries [47.

Interestingly, these constraints also arise naturally from the relationship between general relativity and $B F$ theory, a 4-dimensional topological field theory [15, 16]. Let us recall how this works in the case of signature ++++ . Suppose we have a smooth 4-manifold $M$ and a vector bundle $\mathcal{T} \rightarrow M$ isomorphic to the tangent bundle and equipped with an orientation and positive-definite metric. There is a formulation of general relativity in which the basic fields are a metric-preserving connection $A$ on $\mathcal{T}$ and a $\mathcal{T}$-valued 1 -form $e$, usually called the 'cotetrad' field. The action is given by

$$
\int_{M} \operatorname{tr}(e \wedge e \wedge F),
$$

where $F$ is the curvature of $A$, the wedge product of $\Lambda \mathcal{T}$-valued forms is taken in the obvious way, and the map $\operatorname{tr}: \Lambda^{4} \mathcal{T} \rightarrow \mathbb{R}$ is defined using the metric and orientation on $\mathcal{T}$. The equations of motion are

$$
d_{A}(e \wedge e)=0, \quad e \wedge F=0,
$$

where $d_{A}$ denotes the exterior covariant derivative. When $e$ is nondegenerate these equations are equivalent to the vacuum Einstein equations.

On the other hand, one can modify this formulation, working not with the cotetrad field $e$ but with the field $E=e \wedge e$. First, note that if one takes $E$ to be an arbitrary $\Lambda^{2} \mathcal{T}$-valued 2-form and uses the action

$$
\int_{M} \operatorname{tr}(E \wedge F)
$$

one obtains not general relativity but $B F$ theory, whose equations of motion are simply

$$
d_{A} E=0, \quad F=0 .
$$

The reason is that not every such $E$ field can be written as $e \wedge e$ for some cotetrad field $e$. For this to hold, it must satisfy some extra constraints. If one finds stationary points of the above action subject to these constraints, one obtains equations equivalent to the vacuum Einstein equations, at least when $E$ is nondegenerate.

Now, doing general relativity with the cotetrad field $e$ is very much like describing 4 -simplices using vectors for edges, while doing general relativity with the $E$ field is very much like describing 4 -simplices using bivectors for faces. Suppose we have a 4-simplex affinely embedded in $\mathbb{R}^{4}$. We can number its vertices $0,1,2,3,4$, and translate it so that the vertex 0 is located at the origin. Then one way to describe its geometry is by the positions $e_{1}, e_{2}, e_{3}, e_{4}$ of the other four vertices. Another way is to use the bivectors

$$
E_{a b}=e_{a} \wedge e_{b} \in \Lambda^{2} \mathbb{R}^{4}
$$


corresponding to the six triangular faces with 0 as one of their vertices. However, not every collection of bivectors $E_{a b}$ comes from a 4-simplex this way. In addition to the obvious skew-symmetry $E_{a b}=-E_{b a}$, some extra constraints must hold. They have exactly the same form as the extra constraints that we need to obtain general relativity from $B F$ theory.

Barrett and Crane showed that these constraints take a particularly nice form if we describe them in terms of bivectors corresponding to all ten triangular faces of the 4-simplex. Quantizing these constraints, one obtains conditions that a $\operatorname{Spin}(4)$ spin foam must satisfy to describe a quantum 4-geometry built from flat quantum 4simplices. Interestingly, these conditions also guarantee that the quantum geometries for 3-dimensional submanifolds obtained using the right-handed copy of $\mathrm{SU}(2)$ agree with those coming from the left-handed copy.

In Section 5 we briefly describe two spin foam models for Euclidean quantum gravity in four dimensions: the recently proposed state sum model of Barrett and Crane (which may dually be formulated as a spin foam model), and a closely related model in which no background spacetime manifold is assumed. This section is only intended as a sketch of a future, more careful investigation of specific spin foam models.

\section{Spin foams}

A spin network is a graph with edges labeled by representations and vertices labeled by intertwining operators, or 'intertwiners' for short. Spin foams are like spin networks, but with everything one dimension higher. There are many choices of what we might take as higher-dimensional analogues of graphs. In decreasing order of generality, some well-known possibilities include CW complexes, piecewise linear cell complexes, combinatorial CW complexes, and simplicial complexes [21, 29, 30, 52]. We shall work with piecewise linear cell complexes, but it is good to keep in mind that there are other alternatives with their own advantages. Also, while one tends to think of 2-dimensional topology as a rather trivial business because 2-manifolds are easy to classify, it is worth noting that there are many deep unsolved problems concerning the various sorts of 2-dimensional complexes, such as Whitehead's asphericity question, the Andrews-Curtis conjecture, and Zeeman's conjecture. In working with spin foams, therefore, we should keep in mind that there is a lot of pre-existing mathematical infrastructure to draw upon, but also some deep questions yet to be understood. The book by Hog-Angeloni, Metzler and Sieradski serves as an excellent overview to all this [29].

In what follows we work with piecewise linear cell complexes as defined by Rourke and Sanderson [52]. The precise definition can be found in Appendix A, but we provide a rough sketch here. The basic notion involved is that of a 'cell'. A 0-cell is simply a point, a 1-cell is a closed interval, a 2-cell is a polygon, and so on. Any cell has certain lower-dimensional cells (together with itself) as 'faces', and we write 
$Y \leq X$ if $Y$ is a face of $X$. A piecewise linear cell complex, or 'complex' for short, is a collection $\kappa$ of cells in $\mathbb{R}^{n}$ such that the intersection of any two cells in $\kappa$ is again in $\kappa$, and the face of any cell in $\kappa$ is again in $\kappa$. In what follows we consider only finite complexes. We write $|\kappa|$ for the subset of $\mathbb{R}^{n}$ consisting of the union of the cells in $\kappa$.

We often need to equip the cells of a complex with orientations. We say a $k$-cell $X$ is oriented if $X$ minus the union of its proper faces, thought of as a $k$-dimensional manifold, is equipped with an orientation, and we say a complex is oriented if each $k$-cell with $k \geq 1$ is oriented. Here we implicitly equip each 0 -cell with its positive orientation. Note that we do not require any consistency between the orientations of the different cells.

To define spin networks we need some notation concerning 1-dimensional oriented complexes. Such a complex has a set $V$ of 0 -cells or vertices, and also a set $E$ of oriented 1-cells or edges. The orientation on each edge $e \in E$ picks out one of its endpoints as its source $s(e) \in V$ and the other as its target $t(e) \in V$. If $v$ is the source of $e$ we say $e$ is outgoing from $v$, while if $v$ is the target of $e$ we say that $e$ is incoming to $v$.

As noted in Smolin's excellent review article [58], one of the virtues of the concept of 'spin network' is its versatility. As a result, no single definition easily captures the whole concept. One can study spin networks either abstractly or embedded in a topological, piecewise linear, smooth or real-analytic manifold. In addition, one can label the spin network edges by representations of either groups or quantum groups. Here we define spin networks for any compact group $G$, which we think of as the gauge group of the physical theory in question [13]. We pick a set of irreducible unitary continuous representations of $G$, one from each equivalence class, to use as labels. Each representation $\rho$ in this set has a dual $\rho^{*}$ in this set. Henceforth when we speak of an irreducible representation of $G$ we always mean one in this set.

Definition 1. A spin network $\Psi$ is a triple $(\gamma, \rho, \iota)$ consisting of:

1. a 1-dimensional oriented complex $\gamma$,

2. a labeling $\rho$ of each edge e of $\gamma$ by an irreducible representation $\rho_{e}$ of $G$,

3. a labeling $\iota$ of each vertex $v$ of $\gamma$ by an intertwiner

$$
\iota_{v}: \rho_{e_{1}} \otimes \cdots \otimes \rho_{e_{n}} \rightarrow \rho_{e_{1}^{\prime}} \otimes \cdots \otimes \rho_{e_{m}^{\prime}}
$$

where $e_{1}, \ldots, e_{n}$ are the edges incoming to $v$ and $e_{1}^{\prime}, \ldots, e_{m}^{\prime}$ are the edges outgoing from $v$.

Now let us go up a dimension and define 'spin foams'. A 2-dimensional oriented complex has a finite set of vertices $V$, a finite set of edges $E$, and finite sets of $n$-sided 2-cells or faces $F_{n}$ for each $n \geq 3$, with only finitely many $F_{n}$ being nonempty. As in a 1-dimensional oriented complex, the orientations of the edges give maps

$$
s, t: E \rightarrow V
$$


assigning to each edge its source and target. In addition, the orientation on any 2-cell $f \in F_{n}$ puts a cyclic ordering on its faces and vertices. Suppose we arbitrarily choose a 'first' vertex for each 2-cell $f$ of our complex. Then we may number all its vertices and edges from 1 to $n$. It is convenient to think of these numbers as lying in $\mathbb{Z}_{n}$.

We thus obtain maps

$$
e_{i}: F_{n} \rightarrow E, \quad v_{i}: F_{n} \rightarrow V \quad i \in \mathbb{Z}_{n}
$$

Note that for each $f \in F_{n}$ either

$$
s\left(e_{i}(f)\right)=v_{i}(f) \text { and } t\left(e_{i}(f)\right)=v_{i+1}(f)
$$

or

$$
t\left(e_{i}(f)\right)=v_{i}(f) \text { and } s\left(e_{i}(f)\right)=v_{i+1}(f) .
$$

If (11) holds, we say $f$ is incoming to $e$, while if (2) holds, we say $f$ is outgoing from $e$. In other words, $f$ is incoming to $e$ if the orientation of $e$ agrees with the orientation it inherits from $f$, while it is outgoing if these orientations do not agree.

First we define a special class of spin foams:

Definition 2. A closed spin foam $F$ is a triple $(\kappa, \rho, \iota)$ consisting of:

1. a 2-dimensional oriented complex $\kappa$,

2. a labeling $\rho$ of each face $f$ of $\kappa$ by an irreducible representation $\rho_{f}$ of $G$,

3. a labeling $\iota$ of each edge $e$ of $\kappa$ by an intertwiner

$$
\iota_{e}: \rho_{f_{1}} \otimes \cdots \otimes \rho_{f_{n}} \rightarrow \rho_{f_{1}^{\prime}} \otimes \cdots \otimes \rho_{f_{m}^{\prime}}
$$

where $f_{1}, \ldots, f_{n}$ are the faces incoming to $e$ and $f_{1}^{\prime}, \ldots, f_{m}^{\prime}$ are the faces outgoing from $e$.

Next we turn to general spin foams. In general, a spin foam $F: \Psi \rightarrow \Psi^{\prime}$ will go from a spin network $\Psi$ to a spin network $\Psi^{\prime}$. It has 'free edges', the edges of the spin networks $\Psi$ and $\Psi^{\prime}$, which are not labeled by intertwiners. It also has edges ending at the spin network vertices, and the intertwiners labeling these edges must match those labeling the spin network vertices. A closed spin foam is just a spin foam of the form $F: \emptyset \rightarrow \emptyset$, where $\emptyset$ is the empty spin network: the spin network with no vertices and no edges.

To make this more precise, suppose $\gamma$ is a 1-dimensional oriented complex and $\kappa$ is a 2 -dimensional oriented complex. Note that the product $\gamma \times[0,1]$ becomes a 2-dimensional oriented complex in a natural way. We say $\gamma$ borders $\kappa$ if there is a one-to-one affine map $c:|\gamma| \times[0,1] \rightarrow|\kappa|$ mapping each cell of $\gamma \times[0,1]$ onto a unique cell of $\kappa$ in an orientation-preserving way, such that $c$ maps $\gamma \times[0,1)$ onto an open subset of $|\kappa|$. Note that in this case, $c$ lets us regard each $j$-cell of $\gamma$ as the face of a 
unique $(j+1)$-cell of $\kappa$. Each vertex $v$ of $\gamma$ is the source or target of a unique edge of $\kappa$, which we denote by $\tilde{v}$, and each edge $e$ of $\gamma$ is the edge of a unique face of $\kappa$, which we denote by $\tilde{e}$.

It is easiest to first define spin foams $F: \emptyset \rightarrow \Psi$ and then deal with the general case:

Definition 3. Suppose that $\Psi=(\gamma, \rho, \iota)$ is a spin network. A spin foam $F: \emptyset \rightarrow \Psi$ is a triple $(\kappa, \tilde{\rho}, \tilde{\iota})$ consisting of:

1. a 2-dimensional oriented complex $\kappa$ such that $\gamma$ borders $\kappa$,

2. a labeling $\tilde{\rho}$ of each face $f$ of $\kappa$ by an irreducible representation $\tilde{\rho}_{f}$ of $G$,

3. a labeling $\tilde{\iota}$ of each edge e of $\kappa$ not lying in $\gamma$ by an intertwiner

$$
\tilde{\iota}_{e}: \rho_{f_{1}} \otimes \cdots \otimes \rho_{f_{n}} \rightarrow \rho_{f_{1}^{\prime}} \otimes \cdots \otimes \rho_{f_{m}^{\prime}}
$$

where $f_{1}, \ldots, f_{n}$ are the faces incoming to $e$ and $f_{1}^{\prime}, \ldots, f_{m}^{\prime}$ are the faces outgoing from e.

such that the following hold:

1. For any edge e of $\gamma, \tilde{\rho}_{\tilde{e}}=\rho_{e}$ if $\tilde{e}$ is incoming to $e$, while $\tilde{\rho}_{\tilde{e}}=\left(\rho_{e}\right)^{*}$ if $\tilde{e}$ is outgoing to e.

2. For any vertex $v$ of $\gamma, \tilde{\iota}_{\tilde{e}}$ equals $\iota_{e}$ after appropriate dualizations.

To define general spin foams, we need the notions of 'dual' and 'tensor product' for spin networks. Suppose $\Psi=(\gamma, \rho, \iota)$ is a spin network. Then the dual of $\Psi$ is the spin network $\Psi^{*}$ with the same underlying 1-dimensional oriented complex $\gamma$, but with each edge $e$ labeled by the representation $\rho_{e}^{*}$, and with each vertex $v$ labeled by the appropriately dualized form of the the intertwining operator $\iota_{v}$.

Suppose that $\Psi=(\gamma, \rho, \iota)$ and $\Psi^{\prime}=\left(\gamma^{\prime}, \rho^{\prime}, \iota^{\prime}\right)$ are disjoint spin networks in the same space $\mathbb{R}^{n}$. Then the tensor product $\Psi \otimes \Psi^{\prime}$ is defined to be the spin network whose underlying 1-dimensional oriented complex is the disjoint union of $\gamma$ and $\gamma^{\prime}$, with edges and vertices labeled by representations and intertwiners using $\rho, \rho^{\prime}$ and $\iota, \iota^{\prime}$.

Definition 4. Given disjoint spin networks $\Psi$ and $\Psi^{\prime}$ in $\mathbb{R}^{n}$ a spin foam $F: \Psi \rightarrow \Psi^{\prime}$ is defined to be a spin foam $F: \emptyset \rightarrow \Psi^{*} \otimes \Psi^{\prime}$.

Our notation here is meant to suggest that there is a category with spin networks as objects and spin foams as morphisms. In other words, we want to be able to compose spin foams $F: \Psi \rightarrow \Psi^{\prime}$ and $F^{\prime}: \Psi^{\prime} \rightarrow \Psi^{\prime \prime}$ and obtain a spin foam $F F^{\prime}: \Psi \rightarrow \Psi^{\prime \prime}$. We want this composition to be associative, and for each spin network $\Psi$ we want a spin foam $1_{\Psi}: \Psi \rightarrow \Psi$ serving as a left and right unit for composition. 
To get this to work, one must deal with some technicalities similar to those that arise when one defines the category of cobordisms in topological quantum field theory [57]. Say one has spin foams $F: \Psi \rightarrow \Psi^{\prime}$ and $F: \Psi^{\prime} \rightarrow \Psi^{\prime \prime}$ with $F=(\kappa, \rho, \iota)$ and $F^{\prime}=\left(\kappa^{\prime}, \rho^{\prime}, \iota^{\prime}\right)$. One would like to define $F F^{\prime}$ to be the result of gluing $\kappa$ to $\kappa^{\prime}$ and labeling the resulting complex with the representations coming from $\rho, \rho^{\prime}$ and the intertwiners coming from $\iota, \iota^{\prime}$. However, there is no guarantee that $\kappa$ and $\kappa^{\prime}$ are disjoint, or that they lie in the same space $\mathbb{R}^{n}$. We can arbitrarily choose disjoint copies of them lying in the same space, but then composition satisfies associativity and the left and right unit laws only up to a certain equivalence relation on spin foams. To get a category, one must therefore take the morphisms to be certain equivalence classes of spin foams. We give the details in Appendix B.

We conclude this section with a few remarks:

1) There is a close analogy between the category of spin foams and the category of cobordisms used in topological quantum field theory. This analogy is heightened if we use the the trivial group as our group $G$. Then a spin network is just a 1-dimensional oriented complex, which we can think of as a model of 'space', and a spin foam is a 2-dimensional oriented complex which we can think of as a 'spacetime'. Using a nontrivial group amounts to equipping these complexes with certain extra labelings, which we can think of as 'fields'.

2) In addition to the 'closed' spin networks as defined above, we could define more general 'open' spin networks with free vertices labeled not by an intertwining operator but by a vector in the representation labeling the incident edge. As noted in the Introduction, open spin networks are closely related to Feynman diagrams. They also arise naturally in the study of gauge theory on a manifold with boundary, where they are embedded in the manifold with their free vertices on the boundary. An example of this is the spin network description of a quantum black hole, where the event horizon plays the role of a boundary [4]. Another example is the spin network description of the asymptotically flat sector of quantum gravity, where the 'boundary' is the sphere at spacelike infinity [60]. To study the dynamics of such situations, it might be useful to introduce spin foams going between open spin networks.

3) We could also define spin networks and spin foams in a more general, purely combinatorial way. Abstracting from the notion of a 1-dimensional oriented complex, one may define a graph to be a pair of finite sets $E, V$ together with maps $s, t: E \rightarrow V$. Every 1-dimensional oriented complex has an underlying graph, and the complex is determined by its graph, at least up to piecewise linear homeomorphism (as explained by Rourke and Sanderson [52]). Conversely, a graph is the underlying graph of a 1dimensional oriented complex if and only if no vertex is both the source and target of the same edge. One can easily generalize the definition of a spin network $\Psi=(\gamma, \rho, \iota)$ by allowing $\gamma$ to be an arbitrary graph.

Similarly, abstracting from the notion of a 2-dimensional oriented complex, one may define a 2-graph to consist of finite sets $V, E$, and $F_{n}$ for $n \geq 3$, with only finitely many $F_{n}$ nonempty, together with maps $s, t: E \rightarrow V$ and $e_{i}: F_{n} \rightarrow E, v_{i}: F_{n} \rightarrow V$ for 
$i \in \mathbb{Z}_{n}$, such that for any $f \in F_{n}$ either (1) or (2) holds. A 2-dimensional oriented complex determines a 2-graph if we arbitrarily choose a 'first' vertex for each face. We can reconstruct the complex from this 2-graph, at least up to piecewise linear homeomorphism [52]. Presumably there are purely combinatorial conditions on a 2graph implying that it comes from a 2-dimensional oriented complex. It is certainly necessary that no vertex should be the source and target of the same edge, and that no edge should be both the $i$ th and $j$ th edge of the same face for $i \neq j$. However, the author does not know necessary and sufficient conditions. In any event, one can generalize the definition of a spin foam $F=(\kappa, \rho, \iota)$ by allowing $\kappa$ to be an arbitrary 2-graph.

4) One could also consider higher-dimensional analogs of spin networks and spin foams. For $B F$ theory and the connection formulation of quantum gravity in three and four dimensions, the basic fields can all be thought of as vector-bundle-valued differential forms of degree $\leq 2$. As we shall see, this suggests that the quantization of these theories should involve only 2-dimensional complexes. Work on ' $p$-branes' and the like suggests that some field theories involving forms of higher degree are classical limits of theories involving higher-dimensional complexes.

\section{Spin foams in lattice gauge theory}

In certain lattice gauge theories, spin networks describe states, while spin foams describe 'histories': the path integral can be computed as a sum over spin foams. In this context we work, not with the abstract spin networks of the previous section, but with spin networks embedded in a manifold representing space. Similarly, we work with spin foams embedded in a manifold representing spacetime. Throughout the rest of the paper we assume these manifolds are compact, oriented, and equipped with a fixed triangulation. The triangulation specifies another decomposition of the manifold into cells called the 'dual complex'. There is a one-to-one correspondence between $k$-simplices in the triangulation of an $n$-manifold and $(n-k)$-cells in the dual complex, each $k$-simplex intersecting its corresponding dual $(n-k)$-cell in a single point. Our spin networks and spin foams live in the appropriate dual complexes, as sketched in the Introduction. We need to work with oriented complexes, so we orient each cell of the dual complex in an arbitrary fashion.

We begin by recalling how spin networks describe states in lattice gauge theory. We fix a compact connected Lie group $G$ as our gauge group, and suppose $S$ is an $(n-1)$-manifold representing 'space'. We assume $S$ is equipped with a triangulation $\Delta$ and a principal $G$-bundle $P \rightarrow S$. Also, we choose a trivialization of $P$ over every 0 -cell of the dual complex $\Delta^{*}$.

The ' $k$-skeleton' of a complex is the subcomplex formed by all cells of dimension less than or equal to $k$. The 1 -skeleton of $\Delta^{*}$ is a graph, and we can set up gauge theory on this graph in the usual way [1]. We represent parallel transport along each 
edge of $\Delta^{*}$ as an element of $G$, so we define the space of connections $\mathcal{A}_{S}$ by

$$
\mathcal{A}_{S}=\prod_{e \in \Delta_{1}^{*}} G
$$

where $\Delta_{k}^{*}$ denotes the set of $k$-cells in $\Delta^{*}$. Similarly, we represent a gauge transform at each vertex of $\Delta^{*}$ as an element of $G$, so we define the group of gauge transformations $\mathcal{G}_{S}$ by

$$
\mathcal{G}_{S}=\prod_{v \in \Delta_{0}^{*}} G
$$

The group $\mathcal{G}_{S}$ acts on $\mathcal{A}_{S}$, and the quotient space $\mathcal{A}_{S} / \mathcal{G}_{S}$ is the space of connections modulo gauge transformations in this setting. The space $\mathcal{A}_{S}$ has a measure on it given by a product of copies of Haar measure, and this measure pushes forward to one on $\mathcal{A}_{S} / \mathcal{G}_{S}$. Using this we define the Hilbert space $L^{2}\left(\mathcal{A}_{S} / \mathcal{G}_{S}\right)$.

Suppose that $\Psi=(\gamma, \rho, \iota)$ is a spin network in $S$, that is, one for which $\gamma$ is the 1skeleton of $\Delta^{*}$. Then by a now familiar argument [13], $\Psi$ defines a state in $L^{2}\left(\mathcal{A}_{S} / \mathcal{G}_{S}\right)$, which we also call $\Psi$. Moreover, such spin network states span $L^{2}\left(\mathcal{A}_{S} / \mathcal{G}_{S}\right)$. In fact, we obtain an orthonormal basis of states as $\rho$ ranges over all labelings of the edges of $\Delta^{*}$ by irreducible representations of $G$ and $\iota$ ranges over all labelings of the vertices by intertwiners chosen from some orthonormal basis.

Next we turn to spin foams. For this, suppose $S$ is a submanifold of some $n$ manifold $M$ representing spacetime. We assume that $P \rightarrow M$ is a principal $G$-bundle over $M$ that restricts to the already given bundle over $S$, and that $\Theta$ is a triangulation of $M$ that restricts to the already given triangulation of $S$. We wish to see how a closed spin foam in $M$ determines a spin network in $S$.

Suppose that $F=(\kappa, \tilde{\rho}, \tilde{\iota})$ is a closed spin foam in $M$, that is, one for which $\kappa$ is the 2-skeleton of $\Theta^{*}$. Note that every $k$-cell $X$ of $\Delta^{*}$ is contained in a unique $(k+1)$-cell $\tilde{X}$ of $\Theta^{*}$. Since $S$ and $M$ are oriented, the normal bundle of $S$ acquires an orientation. Similarly, since $X$ and $\tilde{X}$ are oriented, the normal bundle of the interior of $X$ in $\tilde{X}$ acquires an orientation. But this latter bundle can be identified with the restriction of the normal bundle of $S$ to the interior of $X$, so we have two different orientations on the same bundle. We say that $\tilde{X}$ is incoming to $X$ if these orientations agree, and outgoing to $X$ if they do not. We thus obtain a spin network in $S$ as follows:

Proposition 5. If $F=(\kappa, \tilde{\rho}, \tilde{\iota})$ is a closed spin foam in $M$, there exists a unique spin network $\left.F\right|_{S}=(\gamma, \rho, \iota)$ in $S$ such that:

1. $\gamma$ is the 1-skeleton of $\Delta^{*}$,

2. for any edge e of $\gamma, \tilde{\rho}_{\tilde{e}}=\rho_{e}$ if $\tilde{e}$ is incoming to $e$, while $\tilde{\rho}_{\tilde{e}}=\left(\rho_{e}\right)^{*}$ if $\tilde{e}$ is outgoing to $e$.

3. If $v$ is a vertex of $\gamma$, then $\tilde{\iota}_{\tilde{e}}$ equals $\iota_{e}$ after appropriate dualizations. 
The proof is trivial but the physical idea is important: a history on spacetime determines a state on any submanifold corresponding to space at a given time.

A simpler version of this idea applies when we have a spin foam in a cobordism $M: S \rightarrow S^{\prime}$. Here we assume that $M$ has a boundary which has been identified with the disjoint union of $S$ and $S^{\prime}$. We assume there is a principal $G$-bundle $P \rightarrow M$ and a triangulation $\Theta$ of $M$ which restricts to triangulations $\Delta$ and $\Delta^{\prime}$ of $S$ and $S^{\prime}$, respectively. The concept of dual complex is a bit subtler for manifolds with boundary: the dual complex $\Theta^{*}$ has one $k$-cell for each $(n-k)$-cell of $\Theta$, but also includes all the cells of $\Delta^{*}$ and $\Delta^{\prime *}$. We fix a trivialization of $P$ at every vertex of $\Theta^{*}$ on the boundary of $M$.

Note that the 1-skeleton of $\Delta^{*} \cup \Delta^{\prime *}$ borders the 2-skeleton of $\Theta^{*}$, in the sense defined in the previous section. We define a spin foam in $M$ to be a spin foam $F: \Psi \rightarrow \Psi^{\prime}$ such that the underlying complex of $F$ is the 2-skeleton of $\Theta, \Psi$ is a spin network in $S$, and $\Psi^{\prime}$ is a spin network in $S^{\prime}$. Note that this reduces to the previous notion of 'spin foam in $M$ ' when the boundary of $M$ is empty.

Any spin foam $F: \Psi \rightarrow \Psi^{\prime}$ in $M$ determines an operator from $L^{2}\left(\mathcal{A}_{S} / \mathcal{G}_{S}\right)$ to $L^{2}\left(\mathcal{A}_{S^{\prime}} / \mathcal{G}_{S^{\prime}}\right)$, which we also denote by $F$, such that

$$
\left\langle\Phi^{\prime}, F \Phi\right\rangle=\left\langle\Phi^{\prime}, \Psi^{\prime}\right\rangle\langle\Psi, \Phi\rangle
$$

for any states $\Phi, \Phi^{\prime}$. It may seem odd to call this operator ' $F$ ', since it depends only on $\Psi$ and $\Psi^{\prime}$, not any other details of the spin foam. The point is that the spin foam $F$ represents a history going from the initial state $\Psi$ to the final state $\Psi^{\prime}$, and the corresponding operator does not depend on the behavior of this history at 'intermediate times', that is, in the interior of $M$.

We call the operator $F$ a spin foam operator. Just as the space of states $L^{2}\left(\mathcal{A}_{S} / \mathcal{G}_{S}\right)$ is spanned by spin network states, every operator from $L^{2}\left(\mathcal{A}_{S} / \mathcal{G}_{S}\right)$ to $L^{2}\left(\mathcal{A}_{S^{\prime}} / \mathcal{G}_{S^{\prime}}\right)$ will be a linear combination of spin foam operators if there is a spin foam $F: \Psi \rightarrow \Psi^{\prime}$ for every pair of spin networks $\Psi$ and $\Psi^{\prime}$. In a wide variety of lattice gauge theories, the time evolution operator can be computed as a sum of spin foam operators, weighted by amplitudes computed as a product of vertex, edge, and face amplitudes. For more on how this works in Yang-Mills theory and $B F$ theory, see the paper by Reisenberger [48] and the references therein. $B F$ theory with gauge group $\mathrm{SU}(2)$ is 3-dimensional Euclidean quantum gravity, and the lattice formulation of this theory is just the Ponzano-Regge model [46]. When the cosmological constant $\Lambda$ is nonzero, one obtains instead the Turaev-Viro model [62], where the quantum group $\mathrm{SU}_{q}(2)$ takes the place of the gauge group $\mathrm{SU}(2)$, with the deformation parameter $q$ being a function of $\Lambda$. In the rest of this paper we concentrate on a more complicated example, namely 4-dimensional Euclidean quantum gravity. 


\section{Spin networks as quantum 3-geometries}

In this section and the next, our goal is to understand how a linear combination of $\mathrm{SU}(2)$ spin foams in a triangulated 4-manifold can endow it with a 'quantum 4geometry'. The basic picture is simple: spin foam faces give area to the triangles they intersect, while spin foam edges give 3-volume to the tetrahedra they intersect. We also suspect that spin foam vertices give 4-volume to the 4-simplices in which they lie. However, the 3-dimensional aspects of this picture are much better understood than the 4-dimensional ones. Thus, before turning to the question of how spin foams represent quantum 4-geometries, let us recall how spin networks represent quantum 3-geometries. Little in this section is completely new: the main task is to put existing ideas together in a coherent picture.

Let $S$ be a compact oriented 3-manifold equipped with a fixed triangulation $\Delta$ and a principal SU(2) bundle $P \rightarrow S$. Starting from this bundle we can construct the space $L^{2}\left(\mathcal{A}_{S} / \mathcal{G}_{S}\right)$ as in the previous section. Let $\Delta_{k}$ stand for the set of $k$-simplices in $\Delta$. Note that the dual complex $\Delta^{*}$ has one 4-valent vertex for every tetrahedron in $\Delta$ and one edge for every triangle in $\Delta$. Given a tetrahedron $T \in \Delta_{3}$, let $\partial_{0} T, \ldots, \partial_{3} T$ denote its faces, or equivalently, the corresponding edges of the dual complex. From the results of the previous section we have:

$$
L^{2}\left(\mathcal{A}_{S} / \mathcal{G}_{S}\right) \cong \bigoplus_{j} \bigotimes_{T \in \Delta_{3}} \operatorname{Inv}\left(j_{\partial_{0} T} \otimes j_{\partial_{1} T} \otimes j_{\partial_{2} T} \otimes j_{\partial_{3} T}\right)
$$

where 'Inv' denotes the subspace of vectors transforming in the trivial representation, and $j$ ranges over all labelings of the triangles of $\Delta$ by spins. Note that to obtain this isomorphism we need to use the fact that every representation of $\mathrm{SU}(2)$ is equivalent to its dual.

The work of Barbieri [18] clarifies the geometrical significance of this description of $L^{2}\left(\mathcal{A}_{S} / \mathcal{G}_{S}\right)$ : the space $\operatorname{Inv}\left(j_{0} \otimes j_{1} \otimes j_{2} \otimes j_{3}\right)$ describes the states of a 'quantum tetrahedron' whose $i$ th face has area $\frac{1}{2} \sqrt{j_{i}\left(j_{i}+1\right)}$. We review this work in what follows, but first, note an important consequence: the above equation describes $L^{2}\left(\mathcal{A}_{S} / \mathcal{G}_{S}\right)$ as the Hilbert space of quantum 3-geometries of the simplicial complex $\Delta$. Labeling the triangles of $\Delta$ by spins fixes the areas of the triangles and determines a space of states consistent with these areas for each tetrahedron in $\Delta$. To obtain $L^{2}\left(\mathcal{A}_{S} / \mathcal{G}_{S}\right)$ we take the tensor product of all these spaces and then the direct sum over all labelings.

To understand Barbieri's work on the quantum tetrahedron, start with a tetrahedron $T$ having vertices $0,1,2,3$. We can think of a tetrahedron in $\mathbb{R}^{3}$ with one vertex at the origin as an affine map from $T$ to $\mathbb{R}^{3}$ sending the vertex 0 to the origin. Such a map is given by fixing vectors $e_{1}, e_{2}, e_{3} \in \mathbb{R}^{3}$ corresponding to the edges 01,02 , and 03. This data is precisely a 'cotriad', a linear map from the tangent space of the vertex 0 of $T$ to $\mathbb{R}^{3}$. In the Palatini approach to general relativity one works with a similar object, namely the 'cotriad field', which can be thought of locally as an $\mathbb{R}^{3}$ valued 1-form $e$. But a crucial idea in modern canonical quantum gravity is to work 
not with $e$ but with the $\Lambda^{2} \mathbb{R}^{3}$-valued 2-form $e \wedge e$, often called the 'densitized cotriad field'. Using this rather than the cotriad field is the reason why areas rather than lengths are the basic geometrical observables in the spin network approach to quantum gravity. This suggests an alternate description of the geometry and orientation of a tetrahedron in terms of faces rather than edges.

In this alternate description, we work not with the vectors $e_{i}$ but with the bivectors $e_{i} \wedge e_{j}$. (A bivector in $n$ dimensions is simply an element of $\Lambda^{2} \mathbb{R}^{n}$; in the presence of a metric a bivector in three dimensions can be identified with an 'axial vector', which in turn can be identified with a vector in the presence of an orientation.) We denote these bivectors by:

$$
E_{1}=e_{2} \wedge e_{3}, \quad E_{2}=e_{3} \wedge e_{1}, \quad E_{3}=e_{1} \wedge e_{2} .
$$

Not every triple of bivectors $E_{i}$ comes from a triple of vectors $e_{i}$ this way. It is necessary and sufficient that either the $E_{i}$ are linearly independent and satisfy

$$
\epsilon_{I J K} E_{1}^{I} E_{2}^{J} E_{3}^{K}>0
$$

or that they are all multiples of a fixed bivector. The first case is the generic one; it occurs whenever the vectors $e_{i}$ are linearly independent. In this case, the $E_{i}$ determine the $e_{i}$ up to a parity transformation $e_{i} \mapsto-e_{i}$. The second case occurs when the vectors $e_{i}$ lie in a plane; in this case the $E_{i}$ determine the $e_{i}$ up to an area-preserving (but possibly orientation-reversing) linear transformation of this plane.

In short, the map from triples $e_{i}$ to triples $E_{i}$ is neither one-to-one nor onto. Barbieri has suggested an interesting way around this, at least in the generic case. This trick involves fixing an orientation on $\mathbb{R}^{3}$. If the linearly independent triple $e_{i}$ is right-handed, we define the $E_{i}$ as above, but if the triple $e_{i}$ is left-handed, we define the $E_{i}$ with a minus sign:

$$
E_{1}=-e_{2} \wedge e_{3}, \quad E_{2}=-e_{3} \wedge e_{1}, \quad E_{3}=-e_{1} \wedge e_{2},
$$

This establishes a one-to-one and onto map from linearly independent triples $e_{i}$ to linearly independent triples $E_{i}$. While somewhat problematic, we tentatively adopt this strategy in what follows, since it allows us the convenience of working with arbitrary triples $E_{i}$ if we simply accept the fact that a linearly dependent triple $E_{i}$ does not describe a unique tetrahedron.

Actually, rather than working directly with a triple of bivectors, it is useful to introduce a fourth:

$$
E_{0}=-E_{1}-E_{2}-E_{3}
$$

Then, if we use a metric and a orientation to think of the $E_{i}$ as vectors, each $E_{i}$ is normal to the $i$ th face of the tetrahedron (the face missing the $i$ th vertex), with magnitude given by the area of that face. We can obtain either inwards or outwards normals this way. In this description, the constraint $E_{0}+E_{1}+E_{2}+E_{3}=0$ is a 
consequence of the fact that the integral of the unit normal to a 2-sphere piecewise linearly embedded in $\mathbb{R}^{3}$ must vanish. We call it the closure constraint, since it says that the faces can close to form a tetrahedron.

To 'quantize' the tetrahedron, Barbieri first quantizes the bivectors $E_{i}$ and then imposes the closure constraint. The notion of 'quantized bivectors' is implicit in Penrose's ground-breaking work on spin networks and 'quantized directions' 41, 43, but quantized bivectors really date back to early quantum mechanics, where they were introduced to describe angular momentum. Classically, the angular momentum is a bivector. Quantum mechanically, the components of the angular momentum no longer commute, but instead satisfy

$$
\left[J^{1}, J^{2}\right]=i J^{3}, \quad\left[J^{2}, J^{3}\right]=i J^{1}, \quad\left[J^{3}, J^{1}\right]=i J^{2} .
$$

The uncertainty principle thus prevents us from simultaneously measuring all 3 components with arbitrary accuracy. Moreover, each component takes on a discrete spectrum of values.

This is all very elementary, but a more sophisticated viewpoint is also helpful. We can use a metric on $\mathbb{R}^{3}$ to identify $\Lambda^{2} \mathbb{R}^{3}$ with $\mathfrak{s o}(3)^{*}$, which is the phase space of a classical spinning point particle. Any element $f \in \mathfrak{s o}(3)$ gives a linear function on $\mathfrak{s o}(3)^{*}$, essentially the angular momentum about a certain axis. The space $\mathfrak{s o}(3)^{*}$ is naturally equipped with the 'Kirillov-Kostant Poisson structure', in which the Poisson brackets of any two such linear functions is given by

$$
\{f, g\}=[f, g] \text {. }
$$

Being odd-dimensional, $\mathfrak{s o}(3)^{*}$ is not a symplectic manifold, but it is foliated by symplectic leaves, namely the concentric spheres about the origin, which are just the orbits of the action of $\mathrm{SO}(3)$ - the so-called 'coadjoint orbits'. Following Kirillov and Kostant, we can apply geometric quantization to this phase space [27. Only the 'integral' coadjoint orbits contribute, that is, those for which symplectic 2-form divided by $2 \pi$ defines an integral cohomology class. These are the spheres of radius $j=0, \frac{1}{2}, 1, \ldots$ Over each such sphere there is a complex line bundle having the symplectic 2-form as its curvature, and if we think of this sphere as the Riemann sphere, the space of holomorphic sections of this line bundle is naturally isomorphic to the spin- $j$ representation of $\mathfrak{s o}(3)$. The direct sum of all these spaces is the Hilbert space of a quantum spinning point particle, or in other words, the Hilbert space of a quantum bivector in three dimensions. We denote this space by

$$
\mathcal{H}=\bigoplus_{j} j
$$

where we denote the spin- $j$ representation of $\mathfrak{s o}(3)$ simply by $j$. This space is a representation of $\mathfrak{s o}(3)$, so there are operators $J^{1}, J^{2}, J^{3}$ on it satisfying the above commutation relations. Note that this space is not a represention of $\mathrm{SO}(3)$, but only 
of its universal cover, $\mathrm{SU}(2)$. In this way quantum bivectors are naturally related to $\mathrm{SU}(2)$ gauge theory.

To construct the Hilbert space of the quantum tetrahedron, Barbieri starts with $\mathcal{H}^{\otimes 4}$, the tensor product of four copies of the Hilbert space for a quantum bivector, one for each face of tetrahedron. On this space we have operators

$$
\begin{aligned}
& \hat{E}_{0}^{I}=J^{I} \otimes 1 \otimes 1 \otimes 1 \\
& \hat{E}_{1}^{I}=1 \otimes J^{I} \otimes 1 \otimes 1 \\
& \hat{E}_{2}^{I}=1 \otimes 1 \otimes J^{I} \otimes 1 \\
& \hat{E}_{3}^{I}=1 \otimes 1 \otimes 1 \otimes J^{I}
\end{aligned}
$$

where $I=1,2,3$. The Hilbert space of the quantum tetrahedron is then defined as the subspace

$$
\mathcal{T}=\left\{\psi \in \mathcal{H}^{\otimes 4}:\left(\hat{E}_{0}+\hat{E}_{1}+\hat{E}_{2}+\hat{E}_{3}\right) \psi=0\right\}
$$

on which the quantized version of the closure constraint holds. Since $\mathcal{H}^{\otimes 4}$ is naturally a representation of $\mathfrak{s o}(3)$ and the components of the closure constraint generate the $\mathfrak{s o}(3)$ action, it follows that

$$
\mathcal{T} \cong \bigoplus_{j_{0}, j_{1}, j_{2}, j_{3}} \operatorname{Inv}\left(j_{0} \otimes j_{1} \otimes j_{2} \otimes j_{3}\right)
$$

Classically the area of the $i$ th face of the tetrahedron is given by

$$
A_{i}=\frac{1}{2}\left(E_{i} \cdot E_{i}\right)^{1 / 2}
$$

Quantizing these expressions, we define area operators on $\mathcal{T}$ by

$$
\hat{A}_{i}=\frac{1}{2}\left(\hat{E}_{i} \cdot \hat{E}_{i}\right)^{1 / 2}
$$

for $i=0,1,2,3$. These operators commute and are simultaneously diagonalized on the subspaces $\operatorname{Inv}\left(j_{0} \otimes \cdots \otimes j_{3}\right)$; the eigenvalue of $\hat{A}_{i}$ on this subspace is $\frac{1}{2} \sqrt{j_{i}\left(j_{i}+1\right)}$. Similarly, the volume $V$ of a tetrahedron is given classically in terms of the bivectors $E_{i}$ by

$$
V=\frac{1}{6}\left|\epsilon_{I J K} E_{1}^{I} E_{2}^{J} E_{3}^{K}\right|^{1 / 2}
$$

so we define the volume operator on $\mathcal{T}$ by

$$
\hat{V}=\frac{1}{6}\left|\epsilon_{I J K} \hat{E}_{1}^{I} \hat{E}_{2}^{J} \hat{E}_{3}^{K}\right|^{1 / 2}
$$

More precisely, this operator is first defined on $\mathcal{H}^{\otimes 4}$, but since it commutes with the action of $\mathfrak{s o}(3)$ on this space it preserves the subspace $\mathcal{T}$. (In the above formulas, we have ignored factors involving $c, \hbar, 8 \pi G$, and the Immirzi parameter [31].) 
It follows that on the space

$$
L^{2}\left(\mathcal{A}_{S} / \mathcal{G}_{S}\right) \cong \bigoplus_{j} \bigotimes_{T \in \Delta_{3}} \operatorname{Inv}\left(j_{\partial_{0} T} \otimes j_{\partial_{1} T} \otimes j_{\partial_{2} T} \otimes j_{\partial_{3} T}\right)
$$

we can define an area operator $\hat{A}_{f}$ for each triangle $f \in \Delta_{2}$ and a volume operator $\hat{V}_{T}$ for each tetrahedron $T \in \Delta_{3}$. All these operators commute, and on the subspace of $L^{2}\left(\mathcal{A}_{S} / \mathcal{G}_{S}\right)$ corresponding to a given labeling $j$, each area operator $\hat{A}_{f}$ has eigenvalue $\frac{1}{2} \sqrt{j_{f}\left(j_{f}+1\right)}$.

The reader may find it curious how imposing the closure constraint automatically restricts attention to rotation-invariant aspects of the geometry of the quantum tetrahedron. In fact, the constraint $E_{0}+\cdots+E_{3}=0$ is a kind of simplicial version of the Gauss constraint $d_{A} E=0$. Since the Gauss constraint generates $\mathrm{SU}(2)$ gauge transformations, it should not be surprising that the closure constraint generates rotations of the tetrahedron. Imposing the closure constraint for a given tetrahedron of $\Delta$ has the same effect as imposing gauge-invariance at the spin network vertex centered at that tetrahedron.

Making this precise requires a bit of care. We cannot obtain $L^{2}\left(\mathcal{A}_{S} / \mathcal{G}_{S}\right)$ simply by starting with a tensor product of copies of $\mathcal{H}$, one for each triangle in $\Delta$, and then imposing a closure constraint for each tetrahedron. This gives too small a space of states. Instead, we must first 'explode' the triangulation $\Delta$ into a disjoint union of tetrahedra. The resulting complex has two triangles for every triangle in $\Delta$. If we take a tensor product of one copy of $\mathcal{H}$ for each triangle in this 'exploded' complex, we obtain the Hilbert space

$$
H(S)=\bigotimes_{T \in \Delta_{3}} \mathcal{H}^{\otimes 4}
$$

On this space we have operators $\hat{E}_{i}^{I}(T)$ generating $\mathfrak{s o}(3)$ actions on each copy of $\mathcal{H}$, one for each face $i=0,1,2,3$ of each tetrahedron $T$ in $\Delta$. These give area operators

$$
\hat{A}_{i}(T)=\frac{1}{2}\left(\hat{E}_{i}(T) \cdot \hat{E}_{i}(T)\right)^{1 / 2} .
$$

Starting from this big Hilbert space we can impose constraints as follows to get down to $L^{2}\left(\mathcal{A}_{S} / \mathcal{G}_{S}\right)$. First, since each triangle in $\Delta$ corresponds to a pair of triangles in the 'exploded' complex, we can impose constraints saying that both triangles in each such pair have the same area. In other words, we can take the subspace of states $\psi \in H(S)$ for which

$$
A_{i}(T) \psi=A_{i^{\prime}}\left(T^{\prime}\right) \psi
$$

whenever $T, T^{\prime}$ are tetrahedra in $\Delta$ with $\partial_{i} T=\partial_{i^{\prime}} T^{\prime}$. We obtain the subspace

$$
\begin{aligned}
\bigoplus_{j} \bigotimes_{T \in \Delta_{3}} j_{\partial_{0} T} \otimes j_{\partial_{1} T} \otimes j_{\partial_{2} T} \otimes j_{\partial_{3} T} & \cong \bigoplus_{j} \bigotimes_{f \in \Delta_{2}} j_{f} \otimes j_{f} \\
& \cong \bigotimes_{f \in \Delta_{2}} L^{2}(S U(2)) \\
& \cong L^{2}\left(\mathcal{A}_{S}\right) .
\end{aligned}
$$


where $j$ ranges over all labelings of triangles in $\Delta$ by spins.

Next, we can impose a copy of the closure constraint for each tetrahedron, picking out states $\psi$ for which

$$
\left(\hat{E}_{0}(T)+\hat{E}_{1}(T)+\hat{E}_{2}(T)+\hat{E}_{3}(T)\right) \psi=0
$$

for all $T$ in $\Delta$. This gives the subspace

$$
\bigoplus_{j} \bigotimes_{T \in \Delta_{3}} \operatorname{Inv}\left(j_{\partial_{0} T} \otimes j_{\partial_{1} T} \otimes j_{\partial_{2} T} \otimes j_{\partial_{3} T}\right) \cong L^{2}\left(\mathcal{A}_{S} / \mathcal{G}_{S}\right)
$$

as desired.

We can also impose these constraints in the other order. If starting from $H(S)$ we impose a copy of the closure constraint for each tetrahedron, we obtain the subspace $\bigotimes_{T \in \Delta_{3}} \mathcal{T}$. Then, imposing equal-area constraints for pairs of triangles in the 'exploded' complex, we obtain $L^{2}\left(\mathcal{A}_{S} / \mathcal{G}_{S}\right)$. In short, the big space $H(S)$ contains both $L^{2}\left(\mathcal{A}_{S}\right)$ and $\bigotimes_{T \in \Delta_{3}} \mathcal{T}$, and the intersection of these two spaces is $L^{2}\left(\mathcal{A}_{S} / \mathcal{G}_{S}\right)$.

We conclude with a few remarks:

1) It is interesting to compare the area and volume operators discussed above with others appearing in the quantum gravity literature. Most previous work has been done (at least implicitly) in the real-analytic rather than the piecewise-linear context. The area and volume operators first introduced by Rovelli and Smolin 56 differ from those later studied by Ashtekar, Lewandowski and collaborators [6, 7, 8, 9, 33]. However, these differences do not arise in situations involving spin networks with generic 4-valent vertices (i.e., those for which no triple of incident edges have linearly dependent tangent vectors). In such situations, their formulas agree with the formulas above up to constant factors.

Loll works in the piecewise-linear context, but she considers 6 -valent spin networks dual to a cubical lattice [34, 35, 36, 37]. If one sets to zero two of the spins labeling the six edges incident to a vertex, her area and volume operators match those above up to constant factors, except for one difference: she does not include an absolute value in the definition of the volume operator. Instead, she notes that the nonzero eigenvalues of the operator $\epsilon_{I J K} \hat{E}_{1}^{I} \hat{E}_{2}^{J} \hat{E}_{3}^{K}$ come in pairs of the same magnitude but opposite sign, and proposes eliminating the subspace on which this operator is negative. As described above, Barbieri's work suggests another way out of this problem: changing the definition of $E_{i}$ in terms of the cotriad field $e_{i}$, so that the above quantity is negative when the cotriad is left-handed. Of course, this strategy cannot be blithely adopted without more study. It would also be worthwhile to better understand the constant factors by which the area and volume operators used here differ from those appearing elsewhere.

2) An alternate approach to the quantum tetrahedron involves imposing the closure constraint at the classical level. Here we start with the product of four copies of $\mathfrak{s o}(3)^{*}$, equipped with its product Poisson structure. This space is foliated by 8 dimensional symplectic leaves of the form $S^{2} \times S^{2} \times S^{2} \times S^{2}$, where the radius $j_{i}$ of 
the $i$ th sphere is the area of the $i$ th face of the tetrahedron. The symplectic structure on a leaf is integral precisely when each $j_{i}$ equals $0, \frac{1}{2}, 1, \ldots$. To impose the closure constraint we apply symplectic reduction to each leaf [27], first taking the subvariety defined by the constraint $E_{0}+\cdots+E_{3}=0$, and then taking the quotient of this subvariety by the $\mathrm{SU}(2)$ action generated by the constraint. The latter step amounts to considering tetrahedra only modulo rotations. When nonempty, the resulting symplectic manifold is generically 2-dimensional, as one would expect, since the geometry of a tetrahedron modulo rotations has two degrees of freedom after the areas of its faces have been fixed. Applying geometric quantization we obtain the Hilbert space $\operatorname{Inv}\left(j_{0} \otimes \cdots \otimes j_{3}\right)$.

3) The space $\operatorname{Inv}\left(j_{0} \otimes j_{1} \otimes j_{2} \otimes j_{3}\right)$ has a concrete description which is well-known from the quantum mechanics of angular momentum. We can think of an element of this space as an intertwiner $\iota: j_{0} \otimes j_{1} \rightarrow j_{2} \otimes j_{3}$. Since any tensor product $j \otimes k$ decomposes as

$$
j \otimes k \cong|j-k| \oplus|j-k|+1 \oplus \cdots \oplus j+k,
$$

there is a basis of $\operatorname{Inv}\left(j_{0} \otimes \cdots \otimes j_{3}\right)$ labeled by spins $j_{4}$ satisfying

$$
\left|j_{0}-j_{1}\right| \leq j_{4} \leq j_{0}+j_{1}, \quad\left|j_{2}-j_{3}\right| \leq j_{4} \leq j_{2}+j_{3}
$$

and

$$
j_{0}+j_{1}+j_{4}, j_{2}+j_{3}+j_{4} \in \mathbb{Z} .
$$

A more geometrical way to think of this is as follows. Classically, the quantity

$$
A_{01}=\frac{1}{4}\left(\left(E_{0}+E_{1}\right) \cdot\left(E_{0}+E_{1}\right)\right)^{1 / 2}
$$

is the area of a parallelogram formed by the midpoints of four edges of the tetrahedron. At the quantum level, the corresponding area operator is

$$
\hat{A}_{01}=\frac{1}{4}\left(\left(\hat{E}_{0}+\hat{E}_{1}\right) \cdot\left(\hat{E}_{0}+\hat{E}_{1}\right)\right)^{1 / 2} .
$$

Since the operators $\hat{E}_{0}^{I}+\hat{E}_{1}^{I}$ generate an $\mathfrak{s o}(3)$ action on the space $j_{0} \otimes \cdots \otimes j_{3}$ and the operator $\hat{A}_{01}$ commutes with the closure constraint, we can find a basis of $\operatorname{Inv}\left(j_{0} \otimes \cdots \otimes j_{3}\right)$ consisting of eigenvectors of $\hat{A}_{01}$, with eigenvalues of the form $\frac{1}{4} \sqrt{j_{4}\left(j_{4}+1\right)}$. This is just the basis described in the previous paragraph.

More generally, if we split the four vertices of the tetrahedron into two pairs $\{i, j\}$ and $\{k, l\}$, the quantity

$$
A_{i j}=\frac{1}{4}\left(\left(E_{i}+E_{j}\right) \cdot\left(E_{i}+E_{j}\right)\right)^{1 / 2}
$$

is the area of the parallelogram whose vertices are the midpoints of the four edges of the tetrahedron other than the edges $i j$ and $k l$. At the quantum level, we may define a corresponding parallelogram area operator

$$
\hat{A}_{i j}=\frac{1}{4}\left(\left(\hat{E}_{i}+\hat{E}_{j}\right) \cdot\left(\hat{E}_{i}+\hat{E}_{j}\right)\right)^{1 / 2}
$$


on the space $\operatorname{Inv}\left(j_{0} \otimes \cdots \otimes j_{3}\right)$. Note that there is really just one parallelogram area operator for each splitting, since the closure constraint implies

$$
\hat{A}_{i j}=\hat{A}_{k l} \text {. }
$$

For any splitting, the eigenvectors of the corresponding parallelogram area operator form a basis of $\operatorname{Inv}\left(j_{0} \otimes \cdots \otimes j_{3}\right)$ as described above. However the operators corresponding to different splittings do not commute. Thus different splittings give different bases. The transformations between these bases are given by $6 j$ symbols in a well-known way.

As a consequence of all this, given a 3-manifold $S$ with a triangulation $\Delta$ and a splitting of each tetrahedron in $\Delta$, there is a basis of $L^{2}\left(\mathcal{A}_{S} / \mathcal{G}_{S}\right)$ given by all labelings of triangles and tetrahedra in $\Delta$ by spins satisfying conditions like those in equations (3) and (4) for each tetrahedron.

The noncommutativity of parallelogram area operators can also be understood in terms of the symplectic approach sketched in remark 2 above. Classically, the geometry of a tetrahedron with faces of fixed area has two degrees of freedom, but these degrees of freedom do not Poisson-commute. Thus states of the quantum tetrahedron with faces of fixed area should be described by only one quantum number. To see these two degrees of freedom explicitly, note that while there are three parallelogram areas $A_{01}, A_{02}$, and $A_{03}$, they satisfy one equation:

$$
A_{01}^{2}+A_{02}^{2}+A_{03}^{2}=A_{0}^{2}+A_{1}^{2}+A_{2}^{2}+A_{3}^{2} .
$$

Interestingly, this relation continues to hold at the quantum level.

4) It would be worthwhile trying to find formulas for other geometrical observables as operators in the above context, such as the total scalar curvature, the total scalar extrinsic curvature, and the Hamiltonian constraint. It would be especially interesting to compare the commutation relations of such operators to those obtained by Thiemann [59] in the real-analytic context.

\section{Spin foams as quantum 4-geometries}

We now turn to the question of how spin foams describe quantum 4-geometries. Let $M$ be a compact piecewise-linear 4-manifold equipped with a triangulation $\Theta$. We also assume there is a principal bundle $\tilde{P} \rightarrow M$ with structure group $\operatorname{Spin}(4)$. Using this bundle we can do lattice gauge theory over $M$ as in Section 2 . In particular, we can consider a $\operatorname{Spin}(4)$ spin foam in $M$ and try to understand it as equipping $M$ with a 'quantum 4-geometry'. We can also do this for any formal linear combination of spin foams. Actually, it seems that only formal linear combinations of spin foams satisfying certain constraints have a fully satisfactory interpretation as quantum 4geometries. Nonetheless we begin by fixing a particular spin foam in $M$ and seeing how far we can get. 
One can understand quite a bit about the geometry of a 4-manifold by studying the geometry of 3-manifolds embedded in it. Thus we begin by considering 3-dimensional submanifolds of $M$. Suppose we have an oriented 3-dimensional piecewise-linear submanifold $S \subseteq M$ with a triangulation $\Delta \subseteq \Theta$. Since the group $\operatorname{Spin}(4)$ is a product of two copies of $\mathrm{SU}(2)$, the bundle $\tilde{P}$ is a product of 'left-handed' and 'right-handed' $\mathrm{SU}(2)$ bundles $P^{+}$and $P^{-}$. This implies that the space $\mathcal{A}_{S}$ of connections on $\left.\tilde{P}\right|_{S}$ can be written as a product

$$
\mathcal{A}_{S}=\mathcal{A}_{S}^{+} \times \mathcal{A}_{S}^{-}
$$

where $\mathcal{A}_{S}^{ \pm}$is the space of connections on $\left.P^{ \pm}\right|_{S}$, and similarly

$$
\mathcal{A}_{S} / \mathcal{G}_{S}=\mathcal{A}_{S}^{+} / \mathcal{G}_{S}^{+} \times \mathcal{A}_{S}^{-} / \mathcal{G}_{S}^{-}
$$

where $\mathcal{G}_{S}^{ \pm}$is the space of gauge transformations on $\left.P^{ \pm}\right|_{S}$. Thus we have

$$
L^{2}\left(\mathcal{A}_{S} / \mathcal{G}_{S}\right) \cong L^{2}\left(\mathcal{A}_{S}^{+} / \mathcal{G}_{S}^{+}\right) \otimes L^{2}\left(\mathcal{A}_{S}^{-} / \mathcal{G}_{S}^{-}\right)
$$

where both the 'left-handed' and 'right-handed' factors in this tensor product are spanned by $\mathrm{SU}(2)$ spin networks in the dual 1-skeleton of $S$. In particular, both factors are isomorphic. In what follows we will arbitrarily choose to favor $L^{2}\left(\mathcal{A}_{S}^{+} / \mathcal{G}_{S}^{+}\right)$ in certain constructions, influenced by the Ashtekar's strategy of quantizing gravity using the left-handed spin connection [1, 2, 3]. However, we show that in the end the physics is independent of this choice.

As in Section 3 we can define various geometrically interesting operators on the left-handed Hilbert space $L^{2}\left(\mathcal{A}_{S}^{+} / \mathcal{G}_{S}^{+}\right)$. We can tensor these with the identity on the right-handed Hilbert space to obtain corresponding operators on $L^{2}\left(\mathcal{A}_{S} / \mathcal{G}_{S}\right)$. This allows us to interpret states in the latter space as quantum 3-geometries. Moreover, as described in Section 2, any spin foam $F$ in $M$ determines a state $\left.F\right|_{S} \in L^{2}\left(\mathcal{A}_{S} / \mathcal{G}_{S}\right)$. Thus spin foams in $M$ determine quantum 3-geometries for $S$.

More concretely, note that a spin foam in $M$ is a labeling of each 2-cell in the dual complex $\Theta^{*}$ by an irreducible representation of $\operatorname{Spin}(4)$ together with a labeling of each 1-cell by an intertwining operator. The 1-cells and 2-cells in the dual complex correspond to tetrahedra and triangles in the triangulation $\Theta$, respectively. Moreover, any irreducible representation of $\operatorname{Spin}(4)$ is of the form $j^{+} \otimes j^{-}$where $j^{+}$and $j^{-}$are representations of the left-handed and right-handed copies of $\mathrm{SU}(2)$. Thus if we pick a splitting of each tetrahedron in $\Theta$, a spin foam in $M$ amounts to a labeling of each triangle and each tetrahedron in $\Theta$ by a pair of spins.

As explained above, given any triangle $f$ in the triangulation of $S$ there is an area operator $\hat{A}_{f}^{+}$on $L^{2}\left(\mathcal{A}_{S} / \mathcal{G}_{S}\right)$ coming from the area operator on the left-handed Hilbert space $L^{2}\left(\mathcal{A}_{S}^{+} / \mathcal{G}_{S}^{+}\right)$. We define the expectation value of the area of $f$ in the spin foam $F$ to be

$$
\left\langle F, \hat{A}_{f}^{+} F\right\rangle=\left\langle\left. F\right|_{S},\left.A_{f}^{+} F\right|_{S}\right\rangle .
$$

Note that any triangle $f$ in $\Theta$ lies in some submanifold $S \subseteq M$ of the form we are considering, and the above quantity is independent of the choice of $S$. Thus the 
expectation value of the area of any triangle in $M$ is well-defined in the quantum 4-geometry described by any spin foam. The space $S$ serves only as a disposable tool for studying the geometry of the spacetime $M$.

Note that we can easily extend the above formula to formal linear combinations of spin foams. This allows us to think of $A_{f}^{+}$as an operator on the space of formal linear combinations of spin foams. More generally, we can define an area operator $\hat{A}_{\Sigma}^{+}$for any 2-dimensional submanifold $\Sigma \subseteq M$ built from triangles in $\Theta$ by adding up the area operators for the triangles it contains.

Similarly, for every tetrahedron $T$ in the triangulation of $S$ there is a 3-volume operator $\hat{V}_{T}^{+}$on $L^{2}\left(\mathcal{A}_{S} / \mathcal{G}_{S}\right)$, and we can define the expectation value of the 3 -volume of $T$ in the spin foam $F$ to be

$$
\left\langle F, \hat{V}_{T}^{+} F\right\rangle=\left\langle\left. F\right|_{S},\left.\hat{V}_{T}^{+} F\right|_{S}\right\rangle .
$$

Again the right-hand side is actually independent of $S$, since it depends only on the data by which $F$ labels the tetrahedron $T$ and its faces. As with the area of a triangle, we can also extend $V_{T}$ to an operator on the space of formal linear combinations of spin foams. More generally, we can define the volume operator $\hat{V}_{S}^{+}$for any 3-dimensional submanifold $S \subseteq M$ built from tetrahedra in $\Theta$ to be the sum of volume operators for the tetrahedra it contains.

Now we turn to the question of when a linear combination of spin foams defines a quantum 4-geometry for which each 4-simplex is 'flat'. For this we consider the special case when our submanifold $S \subseteq M$ is the boundary of a single 4-simplex $X$ in the triangulation of $M$. Any state in $L^{2}\left(\mathcal{A}_{S} / \mathcal{G}_{S}\right)$ describes a quantum 3-geometry for $S$, but as explained in the Introduction, we only expect states satisfying certain constraints to describe quantum 3-geometries for which $S$ can be regarded as the boundary of a 'flat' quantum 4-simplex. Following ideas of Barrett and Crane [19], we now describe the subspace

$$
\mathcal{X} \subset L^{2}\left(\mathcal{A}_{S} / \mathcal{G}_{S}\right)
$$

of states with this property. We call $\mathcal{X}$ the 'Hilbert space of the quantum 4 -simplex'. We regard a linear combination of spin foams $\sum c_{i} F_{i}$ as describing a quantum 4geometry for which $X$ is flat when the state $\left.\sum c_{i} F_{i}\right|_{S}$ lies in this subspace.

To obtain the subspace $\mathcal{X}$, we begin by studying the geometry of an ordinary classical 4-simplex in Euclidean $\mathbb{R}^{4}$. Let $S$ be a 4 -simplex with vertices $0,1,2,3,4$. We can think of a 4-simplex in $\mathbb{R}^{4}$ with one vertex at the origin as an affine map from $S$ to $\mathbb{R}^{4}$ sending the vertex 0 to the origin. Such a map is given by fixing vectors $e_{1}, e_{2}, e_{3}, e_{4} \in \mathbb{R}^{4}$ corresponding to the edges $01,02,03$ and 04, as in Figure 2. This data amounts to a 'cotetrad', a linear map from the tangent space of the vertex 0 of $S$ to $\mathbb{R}^{4}$. 


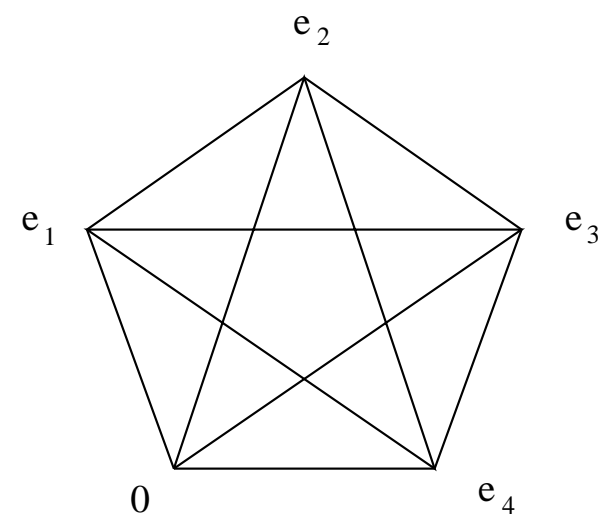

2. The 4-simplex as a cotetrad

Alternatively, we can describe the 4-simplex by associating bivectors to its triangular faces. For any pair $a, b=1,2,3,4$ we can define a bivector

$$
E_{a b}=e_{a} \wedge e_{b}
$$

corresponding to the face $0 a b$. However, not every collection of bivectors $E_{a b}$ is of the form $e_{a} \wedge e_{b}$ for some vectors $e_{a} \in \mathbb{R}^{4}$. In addition to the obvious antisymmetry in the indices $a$ and $b$, the following constraints are also necessary:

$$
E_{a b} \wedge E_{c d}=0 \text { if }\{a, b\} \cap\{c, d\} \neq \emptyset
$$

and

$$
E_{12} \wedge E_{34}=E_{13} \wedge E_{42}=E_{14} \wedge E_{23}
$$

A naive count of degrees of freedom suggests that these conditions might be sufficient: it takes 36 numbers to specify the bivectors $E_{a b}$, but there are 18 independent conditions of type (5) and 2 of type (6), leaving us 16 degrees of freedom, exactly the number of degrees of freedom in the vectors $e_{a}$. Unfortunately, these conditions are not quite enough. In the generic case, when the $E_{a b}(a<b)$ are linearly independent, it can be shown [20, 22] that conditions (5) and (6) admit exactly three sorts of solutions in addition to those of the form

$$
E_{a b}=e_{a} \wedge e_{b}
$$

for some basis $e_{a}$ of $\mathbb{R}^{4}$. The first is to take

$$
E_{a b}=-e_{a} \wedge e_{b}
$$

for some basis $e_{a}$, the second is to take

$$
E_{a b}=\sum_{a, b=1}^{4} \epsilon_{a b c d} e_{c} \wedge e_{d}
$$


and the third is to take

$$
E_{a b}=-\sum_{a, b=1}^{4} \epsilon_{a b c d} e_{c} \wedge e_{d} .
$$

If $E_{a b}$ can be written in any of these four ways, it can be written so uniquely up to a parity transformation $e_{a} \mapsto-e_{a}$. In what follows, we simply ignore these subtleties and take any collection of bivectors $E_{a b}$ antisymmetric in $a, b$ and satisfying (5) and (6) as an adequate substitute for a cotetrad. A more careful treatment would have to address these subtle problems, which are evidently related to those already discussed for tetrahedra in three dimensions.

Barrett and Crane make the all-important observation that conditions (5) and (6) can be rewritten in a simpler way if we use bivectors for all triangular faces of the 4 -simplex, not just those having 0 as a vertex. For this, it is convenient to set $e_{0}=0$ and define

$$
E_{a b c}=\left(e_{c}-e_{b}\right) \wedge\left(e_{b}-e_{a}\right) .
$$

The bivector $E_{a b c}$ corresponds to the triangular face $a b c$; in particular, we have $E_{0 b c}=E_{b c}$. The bivectors $E_{a b c}$ satisfy three sorts of constraints. First, $E_{a b c}$ is totally antisymmetric in the indices $a, b, c$. Second, the bivectors $E_{a b c}(0 \leq a<b<c \leq 4)$ satisfy five closure constraints of the form:

$$
E_{a b c}-E_{a b d}+E_{a c d}-E_{b c d}=0 \quad a<b<c<d
$$

one for each tetrahedral face of $S$. Using these linear constraints, conditions (5) and (6) can be rewritten as the quadratic constraints

$$
E_{a b c} \wedge E_{a^{\prime} b^{\prime} c^{\prime}}=0
$$

which hold whenever the triangles $a b c$ and $a^{\prime} b^{\prime} c^{\prime}$ share at least one edge - that is, when they either share one edge or are the same. The quadratic constraints $E_{a b c} \wedge E_{a b c}=0$ have a particularly nice geometric interpretation: they say the bivectors $E_{a b c}$ can be written as wedge products of vectors in $\mathbb{R}^{4}$.

Before attempting to quantize these constraints, we begin by quantizing a single bivector in 4 dimensions, copying the procedure of Section 3 . Using a metric on $\mathbb{R}^{4}$ we identify $\Lambda^{2} \mathbb{R}^{4}$ with $\mathfrak{s o}(4)^{*}$, which as the dual of a Lie algebra can be thought of as a classical phase space equipped with the Kirillov-Kostant Poisson structure. Since $\mathfrak{s o}(4)$ is isomorphic to $\mathfrak{s o}(3) \oplus \mathfrak{s o}(3)$, when we quantize this phase space we obtain the Hilbert space of a quantum bivector in four dimensions,

$$
\mathcal{H} \otimes \mathcal{H} \cong \bigoplus_{j^{+}, j^{-}} j^{+} \otimes j^{-}
$$

Note that this space is not a representation of $\mathrm{SO}(4)$, but only of its universal cover, $\operatorname{Spin}(4)$. 
In what follows we write the Hilbert space of a quantum bivector in four dimensions as $\mathcal{H}^{+} \otimes \mathcal{H}^{-}$to distinguish between the 'left-handed' and 'right-handed' factors. In fact, these correspond to the self-dual and anti-self-dual parts of the quantum bivector, either of which can be identified with a quantum bivector in three dimensions. To see this, recall that given a metric and orientation on $\mathbb{R}^{4}$, we may define the Hodge star operator

$$
*: \Lambda^{2} \mathbb{R}^{4} \rightarrow \Lambda^{2} \mathbb{R}^{4}
$$

The +1 and -1 eigenspaces of this operator are called the spaces of self-dual and anti-self-dual bivectors, respectively:

$$
\Lambda^{2} \mathbb{R}^{4}=\Lambda_{+}^{2} \mathbb{R}^{4} \oplus \Lambda_{-}^{2} \mathbb{R}^{4} .
$$

This allows us to decompose a bivector $E$ in 4 dimensions into a self-dual part $E^{+}$ and an anti-self-dual part $E^{-}$. Under the isomorphism between $\Lambda^{2} \mathbb{R}^{4}$ and $\mathfrak{s o}(4)^{*}$, this splitting corresponds to the splitting

$$
\mathfrak{s o}(4)^{*} \cong \mathfrak{s o}(3)^{*} \oplus \mathfrak{s o}(3)^{*} .
$$

Thus when we quantize $\mathfrak{s o}(4)^{*}$ with its Kirillov-Kostant Poisson structure, we get a tensor product of Hilbert spaces corresponding to the self-dual and anti-self-dual parts of the space of bivectors.

Now we turn to quantizing the constraints that say when ten bivectors correspond to the faces of a flat 4-simplex. We would like to interpret the closure constraints as imposing gauge-invariance. The closure constraints in equation (17) have minus signs, unlike the closure constraint in our treatment of the quantum tetrahedron. But the reason for these signs is obvious: in our definition of the $E_{a b c}$, we implicitly chose orientations so that two triangular faces of each tetrahedron are oriented clockwise and two are oriented counterclockwise. As in Section 3, the solution we adopt here is to explode $\Delta$ into a disjoint union of five tetrahedra, thus doubling the number of triangles. We can then define a bivector for each face, orienting all the faces counterclockwise. This amounts to working with 20 bivectors $E_{i}(T)$, where $T$ ranges over the five tetrahedra in $\Delta$ and $i=0,1,2,3$ labels the faces of each tetrahedron. In these terms the closure constraints become

$$
E_{0}(T)+E_{1}(T)+E_{2}(T)+E_{3}(T)=0 .
$$

To quantize the closure constraints this way, we start with a Hilbert space

$$
\bigotimes_{T \in \Delta_{3}}\left(\mathcal{H}^{+} \otimes \mathcal{H}^{-}\right)^{\otimes 4}
$$

describing one quantum bivector for each face of the exploded complex. On this space there are operators $E_{i}^{p q}(T)(1 \leq p, q \leq 4)$ generating one $\mathfrak{s o}(4)$ action for each face $i=0,1,2,3$ of each tetrahedron $T$. Then we pick out states $\psi$ for which

$$
\left(\hat{E}_{0}(T)+\hat{E}_{1}(T)+\hat{E}_{2}(T)+\hat{E}_{3}(T)\right) \psi=0
$$


for all $T$ in $\Delta$. By the results of the previous section, this gives the subspace

$$
\bigotimes_{T \in \Delta_{3}} \mathcal{T}^{+} \otimes \mathcal{T}^{-}
$$

where $\mathcal{T}^{+}$and $\mathcal{T}^{-}$are left-handed and right-handed copies of the Hilbert space for the quantum tetrahedron introduced in Section 3 .

Next, again motivated by Section 3, we impose left-handed and right-handed versions of the equal-area constraints for pairs of triangles in the exploded complex that came from the same triangle in $\Delta$. We use the splitting of $\mathfrak{s o}(4)$ into left-handed and right-handed copies of $\mathfrak{s o}(3)$ to write

$$
\hat{E}_{i}(T)=\hat{E}_{i}^{+}(T)+\hat{E}_{i}^{-}(T),
$$

and define left-handed and right-handed area operators by

$$
\hat{A}_{i}^{ \pm}(T)=\frac{1}{2}\left(\hat{E}_{i}^{ \pm}(T) \cdot \hat{E}_{i}^{ \pm}(T)\right)^{1 / 2} .
$$

We then pick out states $\psi$ such that

$$
A_{i}^{+}(T) \psi=A_{i^{\prime}}^{+}\left(T^{\prime}\right) \psi
$$

and

$$
A_{i}^{-}(T) \psi=A_{i^{\prime}}^{-}\left(T^{\prime}\right) \psi
$$

whenever $T, T^{\prime}$ are tetrahedra in $\Delta$ with $\partial_{i} T=\partial_{i^{\prime}} T^{\prime}$. This gives the smaller subspace

$$
\bigoplus_{\rho} \bigotimes_{T \in \Delta_{3}} \operatorname{Inv}\left(\rho_{\partial_{0} T} \otimes \rho_{\partial_{1} T} \otimes \rho_{\partial_{2} T} \otimes \rho_{\partial_{3} T}\right) \cong L^{2}\left(\mathcal{A}_{S} / \mathcal{G}_{S}\right)
$$

where $\rho$ ranges over all labelings of triangles in $\Delta$ by representations of $\operatorname{Spin}(4)$, or in other words, pairs of spins.

Finally we impose the quadratic constraints in the following quantized form:

$$
\left(\hat{E}_{i}(T) \wedge \hat{E}_{i^{\prime}}(T)\right) \psi=0
$$

States satisfying these constraints form Hilbert space of the quantum 4-simplex,

$$
\mathcal{X}=\left\{\psi \in L^{2}\left(\mathcal{A}_{S} / \mathcal{G}_{S}\right):\left(\hat{E}_{i}(T) \wedge \hat{E}_{i^{\prime}}(T)\right) \psi=0 \text { for all } i, i^{\prime}, T\right\} .
$$

Barrett and Crane come close to explicitly describing this space. The key is to rewrite equation (9) as follows:

$$
\left(\hat{E}_{i}^{+}(T) \cdot \hat{E}_{i^{\prime}}^{+}(T)\right) \psi=\left(\hat{E}_{i}^{-}(T) \cdot \hat{E}_{i^{\prime}}^{-}(T)\right) \psi .
$$


One can explicitly solve these constraints in the case $i=i^{\prime}$. Note that a quantum bivector $\psi \in \mathcal{H}^{+} \otimes \mathcal{H}^{-}$satisfies

$$
\left(\hat{E}^{+} \cdot \hat{E}^{+}\right) \psi=\left(\hat{E}^{-} \cdot \hat{E}^{-}\right) \psi
$$

if and only it lies in the subspace

$$
\bigoplus_{j} j \otimes j \subseteq \mathcal{H}^{+} \otimes \mathcal{H}^{-} .
$$

By the same reasoning, the states in $L^{2}\left(\mathcal{A}_{S} / \mathcal{G}_{S}\right)$ satisfying (9) for $i=i^{\prime}$ are precisely those lying in

$$
\bigoplus_{\rho} \bigotimes_{T \in \Delta_{3}} \operatorname{Inv}\left(\rho_{\partial_{0} T} \otimes \rho_{\partial_{1} T} \otimes \rho_{\partial_{2} T} \otimes \rho_{\partial_{3} T}\right)
$$

where $\rho$ ranges only over labelings of triangles in $\Delta$ by representations of the form $j \otimes j$.

The case $i \neq i^{\prime}$ is harder. Note that for $i=i^{\prime}$, we could also write equation (9) in terms of the left-handed and right-handed area operators as follows:

$$
\hat{A}_{i}^{+}(T) \psi=\hat{A}_{i}^{-}(T) \psi
$$

Similarly, we can express the quadratic constraints for $i \neq i^{\prime}$ in terms of left-handed and right-handed versions of the parallelogram area operators described in remark 3 of Section 3:

$$
\hat{A}_{i i^{\prime}}^{ \pm}(T)=\frac{1}{4}\left(\left(\hat{E}_{i}^{ \pm}(T)+\hat{E}_{i^{\prime}}^{ \pm}(T)\right) \cdot\left(\hat{E}_{i}^{ \pm}(T)+\hat{E}_{i^{\prime}}^{ \pm}(T)\right)^{1 / 2} .\right.
$$

Namely, having already imposed (9) for $i=i^{\prime}$, the constraint for $i \neq i^{\prime}$ is equivalent to

$$
\hat{A}_{i i^{\prime}}^{+}(T) \psi=\hat{A}_{i i^{\prime}}^{-}(T) \psi
$$

The general solution of these equations is not yet known, but Barrett and Crane exhibit one solution for each labeling of triangles of $\Delta$ by spins. Thus the Hilbert space $\mathcal{X}$ of a quantum 4 -simplex is an infinite-dimensional subspace of $L^{2}\left(\mathcal{A}_{S} / \mathcal{G}_{S}\right)$.

Note that the quadratic constraints guarantee that all left-handed area operators equal the corresponding right-handed operators when restricted to $\mathcal{X}$. In fact, one can show that vectors in $\mathcal{X}$ are invariant under the transformation

$$
P: L^{2}\left(\mathcal{A}_{S} / \mathcal{G}_{S}\right) \rightarrow L^{2}\left(\mathcal{A}_{S} / \mathcal{G}_{S}\right)
$$

sending each vector $\psi^{+} \otimes \psi^{-}$in $L^{2}\left(\mathcal{A}_{S}^{+} / \mathcal{G}_{S}^{+}\right) \otimes L^{2}\left(\mathcal{A}_{S}^{+} / \mathcal{G}_{S}^{-}\right)$to

$$
P\left(\psi^{+} \otimes \psi^{-}\right)=\psi^{-} \otimes \psi^{+} \text {. }
$$

Thus the left-handed and right-handed versions of all nonchiral geometrical operators - e.g., volume operators - agree on states in $\mathcal{X}$. 
We conclude with some miscellaneous remarks:

1) The spin foam description of quantum 4-geometries is a natural outgrowth of work on 'spinors and spacetime' 44]. This work applies not only to Riemannian geometry but also to Lorentzian geometry. It would thus be of great interest to study spin foams in the Lorentzian context. One will clearly need to use the well-known relationships between $\mathrm{SU}(2), \mathrm{SL}(2, \mathbb{C})$ and $\mathrm{SO}(3,1)$.

2) It would be worthwhile to study the extent to which the role played by simplices in this section and the last can be generalized to cells of other shapes. This is of physical interest because only very special spin networks or spin foams lie in the complex dual to a triangulation. In a physical theory, we should not impose this condition on our spin networks and spin foams without a good physical reason.

3) The possibility of a purely left-handed description of quantum 4-geometries is of great interest, because most of the work relating $B F$ theory to general relativity has focused on the left-handed approach [15, 22, 23, 45, 50]. For this it would be most helpful to find a natural way to intepret $\mathcal{X}$ as a subspace of the left-handed space $L^{2}\left(\mathcal{A}_{S}^{+} / \mathcal{G}_{S}^{+}\right)$. This should be particularly easy if the states of $\mathcal{X}$ found by Barrett and Crane are the only ones. If such an interpretation exists, it might amount to a quantization of the so-called 'Capovilla-Dell-Jacobson' constraints which give general relativity when added to $B F$ theory with gauge group $\mathrm{SU}(2)$.

4) Bivectors $E$ with $E \wedge E=0$ are called simple; these are precisely the bivectors that can be written as a wedge product of two vectors in $\mathbb{R}^{4}$. We may thus call $\bigoplus_{j} j \otimes j$ the Hilbert space of a simple quantum bivector. By the Peter-Weyl theorem, this space is isomorphic to $L^{2}(\mathrm{SU}(2))$. This 'coincidence' deserves to be better understood, because $L^{2}(\mathrm{SU}(2))$ plays a major role in the spin network representation of general relativity formulated as a theory of a left-handed spin connection [5, 14]. It is probably important that both the proof that $\mathrm{SU}(2) \times \mathrm{SU}(2)=\mathrm{Spin}(4)$ and the proof of the Peter-Weyl theorem rely on the left and right actions of $\mathrm{SU}(2)$ on itself.

\section{$5 \quad$ Spin foam models}

To actually extract new physical predictions about quantum gravity from the spin foam formalism is likely to require new ideas and new tools going far beyond those discussed in this paper. In what follows we merely give a taste of what this might be like. In particular, we sketch two spin foam models for 4-dimensional Euclidean quantum gravity. The first model is just another way of looking at the state sum model of Barrett and Crane [19. In this model one treats spacetime as a 4-manifold with a fixed triangulation and computes a discretized path integral as a sum over all Spin(4) spin foams in the dual complex, weighted by amplitudes computed as a product of face, edge, and vertex amplitudes.

The spacetime manifold plays a rather small role in this sort of theory. It does not affect the computation of spin foam amplitudes; it only constrains the set of spin foams being considered, via the requirement that they live in the dual complex. 
This suggests a second model where spin foams are taken as fundamental and no reference is made to an ambient manifold. In this 'abstract' spin foam model, spin foam amplitudes are computed as before. We simply drop the constraint that the spin foams live in the dual complex of a fixed 4-manifold. This model is a crude attempt to combine Penrose's [41, 13] idea of building space from spin networks with Wheeler's [63] vision of spacetime as a kind of 'foam' of vacuum fluctuations corresponding roughly to manifolds with different topologies, but actually built from some 'pregeometry' of a more combinatorial nature.

\section{Quantum gravity on a spacetime with fixed triangulation}

The state sum model of Barrett and Crane is based on the observation that the Ooguri state sum model 42 is a discrete formulation of $B F$ theory, together with the observation that general relativity can be viewed as $B F$ theory with extra constraints forcing the $E$ field to be of the form $e \wedge e$ for some cotetrad field. As we have seen, the quantized version of these constraints is exactly what guarantees that a spin foam represents a reasonable quantum 4-geometry - i.e., one for which every quantum 4simplex is flat, and the left-handed and right-handed 3-geometries of any submanifold coincide. Barrett and Crane obtain their state sum model by taking the Ooguri model and imposing these constraints in their quantized form.

The Ooguri model makes sense for any compact Lie group $G$ and any compact oriented 4-manifold $M$ equipped with a principal $G$-bundle $P \rightarrow M$ and a triangulation $\Theta$. It can be viewed as a rule to compute an amplitude for any spin foam $F=(\kappa, \rho, \iota)$ in $M$. Here $\kappa$ is the 2 -skeleton of the dual complex $\Theta^{*}$, and in what follows we assume the labelings $\iota$ are taken from an orthonormal basis of intertwiners at each vertex. The amplitude is computed as a product of amplitudes for all faces, edges and vertices of the dual complex.

To compute the amplitude for a vertex $v$, note that the spin foam $F$ gives a spin network state $\left.F\right|_{S} \in L^{2}\left(\mathcal{A}_{S} / \mathcal{G}_{S}\right)$ where $S$ is the boundary of the 4-simplex in $\Theta$ containing $v$. To emphasize the dependence on $v$ we write $\left.F\right|_{S}$ as $F_{v}$. In fact, $F_{v}$ is a continuous function on $\mathcal{A}_{S} / \mathcal{G}_{S}$. There is a linear functional $\mu$ on the space of continuous functions on $\mathcal{A}_{S} / \mathcal{G}_{S}$ given by evaluation on the equivalence class of the flat connection 15. We define the amplitude of the vertex $v$ to be $\mu\left(F_{v}\right)$. To compute the edge amplitudes, note that the intertwiner $\iota_{e}$ can be attached to its adjoint $\iota_{e}^{*}$ to form a spin network in the 3 -sphere, which we denote by $\iota_{e} \iota_{e}^{*}$. We define the amplitude for the edge $e$ to be $1 / \mu\left(\iota_{e} \iota_{e}^{*}\right)$, where $\mu$ stands for evaluation on the flat connection on the 3 -sphere. Finally, the amplitude for each face $f$ is simply the dimension of the representation $\rho_{f}$ labeling that face.

For more details on computing face, edge and vertex amplitudes, see the work of Ooguri [42] and also that of Crane, Kauffman and Yetter [25]. We warn the reader that our normalizations differ from theirs slightly, and our notation much more so. To make the connection, it is worth noting that for $\mathrm{SU}(2)$ spin networks on the 3- 
sphere, evaluation on the flat connection is the same as the Penrose evaluation, up to certain pesky but important signs. In particular, what we call $1 / \mu\left(\iota_{e} \iota_{e}^{*}\right)$ is expressed by Crane, Kauffman and Yetter in terms of two ' $\theta$ evaluations', and what we call $\mu\left(F_{v}\right)$ is expressed by them using a ' $15 j$ symbol'.

To obtain their model, Barrett and Crane take $G=\operatorname{Spin}(4)$ and use the same face and edge amplitudes as in the Ooguri model, while modifying the vertex amplitudes. They take as their vertex amplitude the quantity $\mu\left(P\left(F_{v}\right)\right)$, where

$$
P: L^{2}\left(\mathcal{A}_{S} / \mathcal{G}_{S}\right) \rightarrow \mathcal{X}
$$

is the projection onto the Hilbert space of the quantum 4-simplex. Since this projection operator appears at every vertex of the dual complex, this modification of the vertex amplitudes has the effect of picking out precisely those linear combinations of spin foams that correspond to reasonable quantum 4-geometries. In this model, the partition function is given formally as a sum over spin foams in $M$ :

$$
Z(M)=\sum_{F \text { in } M} Z(F)
$$

where the amplitude $Z(F)$ of each spin foam $F=(\kappa, \rho, \iota)$ in $M$ is computed as follows:

$$
Z(F)=\prod_{f \in \kappa_{2}} \operatorname{dim}\left(\rho_{f}\right) \prod_{e \in \kappa_{1}} \frac{1}{\mu\left(\iota_{e} \iota_{e}^{*}\right)} \prod_{v \in \kappa_{0}} \mu\left(P\left(F_{v}\right)\right) .
$$

However, because there are usually infinitely many spin foams in $M$, this sum is likely to diverge. Similarly, if one has a triangulated 4-dimensional cobordism $M: S \rightarrow S^{\prime}$ equipped with a Spin(4)-bundle, one can formally define the time evolution operator

$$
Z(M): L^{2}\left(\mathcal{A}_{S} / \mathcal{G}_{S}\right) \rightarrow L^{2}\left(\mathcal{A}_{S^{\prime}} / \mathcal{G}_{S^{\prime}}\right)
$$

as a sum over all spin foams in $M$ of the corresponding spin foam operators

$$
F: L^{2}\left(\mathcal{A}_{S} / \mathcal{G}_{S}\right) \rightarrow L^{2}\left(\mathcal{A}_{S^{\prime}} / \mathcal{G}_{S^{\prime}}\right)
$$

weighted by appropriately defined amplitudes. But again, in practice this sum is likely to diverge.

The reason for these problems is that $\operatorname{Spin}(4)$ has infinitely many irreducible representations. Barrett and Crane also consider a $q$-deformed variant of their theory. Since quantum groups have only finitely many irreducible representations when $q$ is a suitable root of unity, one obtains well-defined partition functions and time evolution operators this way. While the physical meaning of this $q$-deformed theory is not completely clear, there is an obvious guess. Previously, Crane and Yetter [26] constructed a $q$-deformed version of the Ooguri model and showed that with suitable normalizations the partition function is not only convergent but also independent of the choice of triangulation of $M$. The author has argued [12] that the Crane-Yetter 
model is a discrete formulation of $B F$ theory with a cosmological term, i.e., with the action given by

$$
\int_{M} \operatorname{tr}\left(E \wedge F+\frac{\Lambda}{12} E \wedge E\right),
$$

where the deformation parameter $q$ is a function of the cosmological constant $\Lambda$. Just as general relativity can be described as a constrained $B F$ theory, so general relativity with nonzero cosmological constant can be described as a constrained $B F$ theory with cosmological term. All this taken together suggests that the $q$-deformed version of Barrett and Crane's state sum is a discretization of Euclidean quantum gravity with cosmological constant. In this regard it is also interesting to note Major and Smolin's work on quantum gravity with cosmological constant using $q$-deformed spin networks [40, the degenerate solutions of general relativity with cosmological constant obtained from $B F$ theory with cosmological term [16], and the recent work of Markopoulou and Smolin [39].

When contemplating these issues it is useful to keep in mind the analogies with 3-dimensional quantum gravity. In three dimensions one has the Ponzano-Regge 46] model based on $\mathrm{SU}(2)$ and the Turaev-Viro [62 model based on the quantum group $\mathrm{SU}_{q}(2)$. The former corresponds to Euclidean quantum gravity with $\Lambda=0$, but is divergent, while the latter is convergent and corresponds to Euclidean quantum gravity with $\Lambda \neq 0$. These models can be formulated either in terms of triangulations or dually in terms of spin foams having a 'special spine' as their underlying 2-dimensional complex. Roughly speaking, a 'special polyhedron' is a 2-dimensional complex with only generic singularities, such as one sees in a foam of soap bubbles, and a 'special spine' of a compact 3-manifold $S$ is a special polyhedron embedded in $S$ whose complement is an open ball. For more on these matters, see the books by Turaev 61 and Hog-Angeloni, Metzler and Sieradski [29].

To apply these analogies to the 4-dimensional case, it helps to note that the CraneYetter model, based on triangulations, is equivalent to Turaev's earlier construction using spin foams having a 'skeleton' as their underlying complex [61]. A 'skeleton' of a compact 4-manifold $M$ is a special polyhedron embedded in $M$ whose complement is an open 4-dimensional handlebody.

\section{Quantum gravity without a background spacetime}

As we have seen, the heart of Barrett and Crane's state sum model is the following formula for the amplitude $Z(F)$ of a spin foam $F=(\kappa, \rho, \iota)$ :

$$
Z(F)=\prod_{f \in \kappa_{2}} \operatorname{dim}\left(\rho_{f}\right) \prod_{e \in \kappa_{1}} \frac{1}{\mu\left(\iota_{e} \iota_{e}^{*}\right)} \prod_{v \in \kappa_{0}} \mu\left(P\left(F_{v}\right)\right)
$$

Since this formula does not depend on the ambient spacetime manifold, but only on the spin foam itself, we may consider it more generally as a formula for evaluating the amplitude of any Spin(4) spin foam with four faces meeting at each edge and with ten 
faces and five edges meeting at each vertex in a pattern dual to that of a 4 -simplex. This gives an 'abstract' spin foam model, where there is no picture of spacetime other than that provided by the spin foam itself. Using the formula described in Section 3 , we may think of each face of $\kappa$ as 'carrying' a certain area, which it would give to any imagined surface it intersected transversely. Similarly, we may think of each edge as carrying a certain volume. It remains a challenge to determine, if possible, the 4-volume associated to any vertex. The main point, however, is that if we think of an abstract spin foam as a kind of quantum 4-geometry, the above formula serves as a rule for computing the amplitude of any such quantum 4-geometry.

There are, of course, very serious problems in extracting physics from such a model. Most obviously, it is difficult to sum over all spin foams. Nonetheless it seems worth investigating such models.

\section{Appendix A: Piecewise linear cell complexes}

We follow the definition of piecewise linear cell complexes given by Rourke and Sanderson [52]. A subset $X \subseteq \mathbb{R}^{n}$ is said to be a polyhedron if every point $x \in X$ has a neighborhood in $X$ of the form

$$
\{\alpha x+\beta y: \alpha, \beta \geq 0, \alpha+\beta=1, y \in Y\}
$$

where $Y \subseteq X$ is compact. A compact convex polyhedron $X$ for which the smallest affine space containing $X$ is of dimension $k$ is called a $k$-cell. The term 'polyhedron' may be somewhat misleading to the uninitiated; for example, $\mathbb{R}^{n}$ is a polyhedron, and any open subset of a polyhedron is a polyhedron. Cells, on the other hand, are more special. For example, every 0-cell is a point, every 1-cell is a compact interval affinely embedded in $\mathbb{R}^{n}$, and every 2 -cell is a convex compact polygon affinely embedded in $\mathbb{R}^{n}$.

The 'vertices' and 'faces' of a cell $X$ are defined as follows. Given a point $x \in X$, let $\langle x, X\rangle$ be the union of lines $L$ through $x$ such that $L \cap X$ is an interval with $x$ in its interior. If there are no such lines, we define $\langle x, X\rangle$ to be $\{x\}$ and call $x$ a vertex of $X$. One can show that $\langle x, X\rangle \cap X$ is a cell, and such a cell is called a face of $X$.

One can show that any cell $X$ has finitely many vertices $v_{i}$ and that $X$ is the convex hull of these vertices, meaning that:

$$
X=\left\{\sum \alpha_{i} v_{i}: \alpha_{i} \geq 0, \sum \alpha_{i}=1\right\}
$$

Similarly, any face of $X$ is the convex hull of some subset of the vertices of $X$. However, not every subset of the vertices of $X$ has a face of $X$ as its convex hull. If the cell $Y$ is a face of $X$ we write $Y \leq X$. This relation is transitive, and if $Y, Y^{\prime} \leq X$ we have $Y \cap Y^{\prime} \leq X$

Finally, one defines a piecewise linear cell complex, or complex for short, to be a collection $\kappa$ of cells in some $\mathbb{R}^{n}$ such that: 
1. If $X \in \kappa$ and $Y \leq X$ then $Y \in \kappa$.

2. If $X, Y \in \kappa$ then $X \cap Y \leq X, Y$.

The union of the cells of a complex $\kappa$ is a polyhedron which we denote by $|\kappa|$.

A complex is $k$-dimensional if it has cells of dimension $k$ but no higher. A subcomplex of a complex $\kappa$ is a subset of $\kappa$ which is again a complex, and the $k$-skeleton of $\kappa$ is the subcomplex consisting of all cells of dimension $k$ or less.

\section{Appendix B: The category of spin foams}

For each compact group $G$ we shall define a category $\mathcal{F}$ of spin foams. It is convenient here to restrict attention to 'nondegenerate' spin networks and spin foams. A spin network is nondegenerate if every vertex is the endpoint of at least one edge and every edge is labeled with a nontrivial irreducible representation of $G$. A spin foam is nondegenerate if every vertex is the endpoint of at least one edge, every edge is the edge of at least one face, and every face is labeled with a nontrivial irreducible representation of $G$.

The objects of the category $\mathcal{F}$ of spin foams are nondegenerate spin networks with $G$ as gauge group. As explained in Section 1, to define morphisms between arbitrary objects of $\mathcal{F}$ we need a way to form a tensor product of spin networks whose underlying complexes may not be disjoint, or may not lie in the same space $\mathbb{R}^{n}$. Thus suppose that $\Psi=(\gamma, \rho, \iota)$ and $\Psi^{\prime}=\left(\gamma^{\prime}, \rho^{\prime}, \iota^{\prime}\right)$ are spin networks in $\mathbb{R}^{n}$ and $\mathbb{R}^{n^{\prime}}$, respectively. Suppose that $k, k^{\prime}>0$ satisfy $n+k=n^{\prime}+k^{\prime}=N$. If we map $\gamma$ and $\gamma^{\prime}$ into $\mathbb{R}^{N}$ using the maps

$$
\left(x_{1}, \ldots, x_{n}\right) \mapsto\left(x_{1}, \ldots, x_{n}, 0, \ldots, 0\right)
$$

and

$$
\left(x_{1}, \ldots, x_{n}\right) \mapsto\left(x_{1}, \ldots, x_{n^{\prime}}, 0, \ldots, 1\right)
$$

respectively, their images are disjoint. We call the union of their images a disjoint union of $\gamma$ and $\gamma^{\prime}$. We define a tensor product $\Psi \otimes \Psi^{\prime}$ to be a spin network whose underlying 1-dimensional oriented complex is a disjoint union of $\gamma$ and $\gamma^{\prime}$, with edges and vertices labeled by representations and intertwiners using $\rho, \rho^{\prime}$ and $\iota, \iota^{\prime}$.

We then define a morphism in $\mathcal{F}$ from $\Psi$ to $\Psi^{\prime}$ to be a certain equivalence class of nondegenerate spin foams $F: \emptyset \rightarrow \Psi^{*} \otimes \Psi^{\prime}$. Two spin foams $F=(\kappa, \rho, \iota)$ and $F^{\prime}=\left(\kappa^{\prime}, \rho^{\prime}, \iota^{\prime}\right)$ are regarded as equivalent if one can be obtained from the other by a sequence of the following moves and their inverses:

1. Affine transformation. Suppose $F$ is a spin foam in $\mathbb{R}^{n}$ and $F^{\prime}$ is a spin foam in $\mathbb{R}^{n^{\prime}}$. Then $F^{\prime}$ is obtained from $F$ by affine transformation if: 
(a) there is a one-to-one affine map $\phi: \mathbb{R}^{n} \rightarrow \mathbb{R}^{n^{\prime}}$ establishing a one-to-one correspondence between cells of $\kappa$ and cells of $\kappa$ and preserving the orientations of all cells;

(b) if $f$ is a face of $\kappa$, then $\rho_{f}=\rho_{\phi(f)}^{\prime}$;

(c) if $e$ is a edge of $\kappa$, then $\iota_{e}=\iota_{\phi(e)}^{\prime}$.

2. Subdivision. Suppose $F$ and $F^{\prime}$ are spin foams in $\mathbb{R}^{n}$. Then $F^{\prime}$ is obtained from $F$ by subdivision if:

(a) the oriented 2-dimensional complex $\kappa^{\prime}$ is obtained from $\kappa$ by subdivision;

(b) if the face $f^{\prime}$ of $\kappa^{\prime}$ is contained in the face $f$ of $\kappa$ then $\rho_{f^{\prime}}^{\prime}=\rho_{f}$;

(c) if the edge $e^{\prime}$ of $\kappa^{\prime}$ is contained in the edge $e$ of $\kappa$ then $\iota_{e^{\prime}}^{\prime}=\iota_{e}$, while if $e^{\prime}$ is the edge of two faces of $\kappa^{\prime}$ contained in the same face $f$ of $\kappa$ then $\iota_{e^{\prime}}^{\prime}=1_{\rho_{f}}$.

3. Orientation reversal. Suppose $F$ and $F^{\prime}$ are spin foams in $\mathbb{R}^{n}$. Then $F^{\prime}$ is obtained from $F$ by orientation reversal if:

(a) the 2-dimensional oriented complexes $\kappa$ and $\kappa^{\prime}$ have the same cells, with possibly differing orientations;

(b) if $f$ is a face of $\kappa$, then $\rho_{f}^{\prime}=\rho_{f}$ if $f$ is given the same orientation in $\kappa$ and $\kappa^{\prime}$, while $\rho_{f}^{\prime}=\left(\rho_{f}\right)^{*}$ if it is given opposite orientations in $\kappa$ and $\kappa^{\prime}$;

(c) if $e$ is a edge of $\kappa$, then $\iota_{e}$ equals $\iota_{\phi(e)}^{\prime}$ after appropriate dualizations.

Here it is worth noting a subtle point. A spin foam $F: \emptyset \rightarrow \Psi^{*} \otimes \Psi^{\prime}$ has been defined as a 2-dimensional oriented complex $\kappa$ bordered by the disjoint union $\gamma \cup \gamma^{\prime}$, together with certain labelings of the edges and faces of $\kappa$. It is important to note that part of the data comprising $F$ is a one-to-one affine map from $\left|\gamma \cup \gamma^{\prime}\right| \times[0,1]$ to $|\kappa|$. We use this 'attaching map' to think of $\gamma$ and $\gamma^{\prime}$ as contained in $\kappa$. When two spin foams are related by affine transformation as in the definition above, their attaching maps are required to be related by the same affine transformation in an obvious way. When two spin foams are related by subdivision or orientation reversal, their attaching maps are required to be the same.

We define composition in $\mathcal{F}$ as follows. Suppose we have morphisms $F: \Psi \rightarrow \Psi^{\prime}$ and $F^{\prime}: \Psi^{\prime} \rightarrow \Psi^{\prime \prime}$. Then we can choose representatives of $F$ and $F^{\prime}$ living in the same space $\mathbb{R}^{n}$ such that the copy of $\Psi^{\prime}=\left(\gamma^{\prime}, \rho^{\prime}, \iota^{\prime}\right)$ contained in $F$ is the same as the copy contained in $F^{\prime}$, and the affine maps $c, c^{\prime}:\left|\gamma^{\prime}\right| \times[0,1] \rightarrow \mathbb{R}^{n}$ by which $\gamma$ borders the underlying complexes of $F$ and $F^{\prime}$ fit together to define a single affine map $f:\left|\gamma^{\prime}\right| \times[-1,1] \rightarrow \mathbb{R}^{n}$. We then define the composite $F F^{\prime}$ to be the spin foam whose underlying complex is the union of those of $F$ and $F^{\prime}$, with faces and edges inheriting their labels from $F$ and $F^{\prime}$, except for the edges of $\gamma^{\prime}$, which are labeled by appropriately dualized forms of identity intertwiners. 
One can check that with this definition $\mathcal{F}$ becomes a category. We conclude with two remarks:

1) The reader may wonder why we take affine transformation, subdivision and orientation reversal as the basic forms of equivalence between spin foams instead of just piecewise-linear homeomorphism and orientation reversal. Indeed, if we were only interested in abstract or piecewise-linearly embedded spin foams, we could use such a simpler notion of equivalence. However, smoothly and real-analytically embedded spin foams are also interesting, and one cannot define a smooth or real-analytic embedding of a surface defined only up to piecewise-linear homeomorphism! The category we propose should be useful for studying smoothly or real-analytically embedded spin foams, as well as piecewise-linearly embedded ones.

2) Recall from remark 2 of Section 1 that, in addition to the 'closed' spin networks we have been using, there exist 'open' spin networks. There should be a 2-category whose objects are finite collections of points labeled by vectors in nontrivial irreducible representations of $G$, whose morphisms are equivalence classes of open spin networks, and whose 2-morphisms are equivalence classes of spin foams going between such open spin networks. Since there are tensor and duality operations on spin networks and spin foams, we expect that this is a monoidal 2-category with duals - in fact, a 'strongly involutory' monoidal 2-category with duals. This may be important in attempts to describe quantum gravity in the language of higher-dimensional algebra [17, 24]. However, we shall not endeavor to make this precise here.

\section{Acknowledgments}

I am grateful to the Center for Gravitational Physics and Geometry at Pennsylvania State University for their hospitality while part of this work was done, and especially thank Roumen Borissov, Kirill Krasnov, Fotini Markopoulou, Carlo Rovelli, Lee Smolin, and Jose Antonio Zapata for many helpful discussions while I was there. I also thank Allan Adler, Robt Bryant, John Barrett, Allen Knutson, Justin Roberts, Michael Reisenberger, and Stephen Sawin for help with 2-dimensional complexes, state sum models, and the quantum 4-simplex. This work was partially supported by NSF grant PHY95-14240.

\section{References}

[1] A. Ashtekar, New variables for classical and quantum gravity. Phys. Rev. Lett. 57 (1986), 2244-2247.

[2] A. Ashtekar and invited contributors, New Perspectives in Canonical Gravity, Bibliopolis, Napoli, Italy, 1988. (Available through the American Institute of Physics; errata available from the Center for Gravitational Physics and Geometry at Pennsylvania State University.) 
[3] A. Ashtekar, Lectures on Non-perturbative Canonical Quantum Gravity, World Scientific, Singapore, 1991.

[4] A. Ashtekar, J. Baez, A. Corichi and K. Krasnov, Quantum geometry and black hole entropy, Phys. Rev. Lett. 80 (1998), 904-907.

[5] A. Ashtekar and J. Lewandowski, Projective techniques and functional integration, Jour. Math. Phys. 36 (1995), 2170-2191.

[6] A. Ashtekar and J. Lewandowski, Differential geometry for spaces of connections via graphs and projective limits, Jour. Geom. Phys. 17 (1995), 191-230.

[7] A. Ashtekar and J. Lewandowski, Quantum theory of geometry I: area operators, Class. Quantum Grav. 14 (1997), A55-A81.

[8] A. Ashtekar and J. Lewandowski, Quantum theory of geometry II: volume operators, preprint available as gr-qc/9711031.

[9] A. Ashtekar, J. Lewandowski, D. Marolf, J. Mourão, and T. Thiemann, Quantization of diffeomorphism invariant theories of connections with local degrees of freedom, $J$. Math. Phys. 36 (1995), 6456-6493.

[10] J. Baez, Strings, loops, knots and gauge fields, in Knots and Quantum Gravity, ed. J. Baez, Oxford U. Press, Oxford, 1994.

[11] J. Baez, Generalized measures in gauge theory, Lett. Math. Phys. 31 (1994), 213-223.

[12] J. Baez, Four-dimensional $B F$ theory as a topological quantum field theory, Lett. Math. Phys. 38 (1996), 129-143.

[13] J. Baez, Spin networks in gauge theory, Adv. Math. 117 (1996), 253-272.

[14] J. Baez, Spin networks in nonperturbative quantum gravity, in The Interface of Knots and Physics, ed. L. Kauffman, American Mathematical Society, Providence, Rhode Island, 1996.

[15] J. Baez, Knots and quantum gravity: progress and prospects, in Proceedings of the Seventh Marcel Grossman Meeting on General Relativity, eds. R. Jantzen and G. Mac Keiser, World Scientific, 1996, also available as gr-qc/9410018.

[16] J. Baez, Degenerate solutions of general relativity from topological field theory, to appear in Commun. Math. Phys., preprint available as gr-qc/9702051.

[17] J. Baez and J. Dolan, Higher-dimensional algebra and topological quantum field theory, Jour. Math. Phys. 36 (1995), 6073-6105.

[18] A. Barbieri, Quantum tetrahedra and simplicial spin networks, preprint available as gr-qc/9707010. 
[19] J. Barrett and L. Crane, Relativistic spin networks and quantum gravity, preprint available as gr-qc/9709028.

[20] R. Bryant, personal communication.

[21] R. Fritsch and R. Piccini, Cellular Structures in Topology, Cambridge U. Press, Cambridge, 1990.

[22] R. Capovilla, J. Dell and T. Jacobson, A pure spin-connection formulation of gravity, Class. Quant. Grav. 8 (1991), 59-74.

[23] R. Capovilla, J. Dell, T. Jacobson, and L. Mason, Self-dual 2-forms and gravity, Class. Quant. Grav. 8 (1991) 41-58.

[24] L. Crane, Clock and category: is quantum gravity algebraic?, Jour. Math. Phys. 36 (1995), 6180-6193.

[25] L. Crane, L. Kauffman and D. Yetter, State-sum invariants of 4-manifolds, I, preprint available as hep-th/9409167.

[26] L. Crane and D. Yetter, A categorical construction of 4d TQFTs, in Quantum Topology, eds. L. Kauffman and R. Baadhio, World Scientific, Singapore, 1993.

[27] V. Guillemin and S. Sternberg, Symplectic Techniques in Mathematical Physics, Cambridge U. Press, Cambridge, 1984.

[28] B. Hasslacher and M. Perry, Spin networks are simplicial quantum gravity, Phys. Lett. B103 (1981), 21-24.

[29] C. Hog-Angeloni, W. Metzler, and A. Sieradski, Two-dimensional Homotopy and Combinatorial Group Theory, London Mathematical Society Lecture Note Series 197, Cambridge U. Press, Cambridge, 1993.

[30] J. Hudson, Piecewise Linear Topology, W. A. Benjamin, Reading, Massachusetts, 1969.

[31] G. Immirzi, Quantum gravity and Regge calculus, to appear in the proceedings of the 2nd meeting on Constrained Dynamics and Quantum Gravity, Santa Margherita Ligure, preprint available as gr-qc/9701052.

[32] J. Iwasaki, A reformulation of the Ponzano-Regge quantum gravity model in terms of surfaces, preprint available as gr-qc/9410010.

[33] J. Lewandowski, Volume and quantizations, Class. Quant. Grav. 14 (1997), 71-76.

[34] R. Loll, The volume operator in discretized quantum gravity, Phys. Rev. Lett. 75 (1995) 3048-3051.

[35] R. Loll, Spectrum of the volume operator in quantum gravity, Nucl. Phys. B460 (1996) 143-154. 
[36] R. Loll, Further results on geometric operators in quantum gravity, Class. Quantum Grav. 14 (1997), 1725-1741.

[37] R. Loll, Imposing $\operatorname{det} E>0$ in discrete quantum gravity, Phys. Lett. B399 (1997), 227-232.

[38] F. Markopoulou, Dual formulation of spin network evolution, preprint available as gr-qc/9704013.

[39] F. Markopoulou and L. Smolin, Quantum geometry with intrinsic local causality, preprint available at gr-qc/9712067.

[40] S. Major and L. Smolin, Quantum deformation of quantum gravity, Nucl. Phys. B473 (1996), 267-290.

[41] J. Moussouris, Quantum models of space-time based on recoupling theory, Ph.D. thesis, Department of Mathematics, Oxford University, 1983.

[42] H. Ooguri, Topological lattice models in four dimensions, Mod. Phys. Lett. A7 (1992) 2799-2810.

[43] R. Penrose, Angular momentum: an approach to combinatorial space-time, in Quantum Theory and Beyond, ed. T. Bastin, Cambridge U. Press, Cambridge, 1971.

[44] R. Penrose and W. Rindler, Spinors and Space-Time, 2 volumes, Cambridge U. Press, Cambridge, 1984-1986.

[45] J. Plebanski, On the separation of Einsteinian substructures, Jour. Math. Phys. 18 (1977) 2511-2520.

[46] G. Ponzano and T. Regge, Semiclassical limit of Racah coefficients, in Spectroscopic and Group Theoretical Methods in Physics, ed. F. Bloch, North-Holland, New York, 1968.

[47] T. Regge, General relativity without coordinates, Nuovo Cimento 19 (1961), 558-571.

[48] M. Reisenberger, Worldsheet formulations of gauge theories and gravity, preprint available as gr-qc/9412035.

[49] M. Reisenberger, New constraints for canonical general relativity, Nucl. Phys. B457 (1995), 643-687.

[50] M. Reisenberger, A left-handed simplicial action for Euclidean general relativity, preprint available as gr-qc/9609002.

[51] M. Reisenberger and C. Rovelli, "Sum over surfaces" form of loop quantum gravity, Phys. Rev. D56 (1997), 3490-3508.

[52] C. Rourke and B. Sanderson, Introduction to Piecewise-Linear Topology, Springer Verlag, Berlin, 1972. 
[53] C. Rovelli, The basis of the Ponzano-Regge-Turaev-Viro-Ooguri model is the loop representation basis, Phys. Rev. D48 (1993), 2702-2707.

[54] C. Rovelli and L. Smolin, Loop representation for quantum general relativity, Nucl. Phys. B331 (1990), 80-152.

[55] C. Rovelli and L. Smolin, Spin networks in quantum gravity, Phys. Rev. D52 (1995), 5743-5759.

[56] C. Rovelli and L. Smolin, Discreteness of area and volume in quantum gravity, Nucl. Phys. B442 (1995), 593-622. Erratum, ibid. B456 (1995), 753.

[57] S. Sawin, Links, quantum groups and TQFTs, in Bull. Amer. Math. Soc. 33 (1996), 413-445.

[58] L. Smolin, The future of spin networks, preprint available as gr-qc/9702030.

[59] T. Thiemann, Anomaly-free formulation of non-perturbative, four-dimensional Lorentzian quantum gravity, Phys. Lett. B380 (1996), 257-264.

[60] T. Thiemann, QSD VI: Quantum Poincaré algebra and a quantum positivity of energy theorem for canonical quantum gravity, preprint available as gr-qc/9705020.

[61] V. Turaev, Quantum Invariants of Knots and 3-Manifolds, de Gruyter, New York, 1994.

[62] V. Turaev and O. Viro, State sum invariants of 3-manifolds and quantum $6 j$ symbols, Topology 31 (1992), 865-902.

[63] J. A. Wheeler, Geometrodynamics, Academic Press, New York, 1962.

[64] J. Zapata, A combinatorial approach to diffeomorphism invariant quantum gauge theories, preprint available as gr-qc/9703037. 